\title{
A bonded joint finite element for a symmetric double lap joint subjected to mechanical and thermal loads
}

\author{
Peter A. Gustafson $\ddagger$ and Anthony M. Waas $*, \dagger, \S$ \\ Department of Aerospace Engineering, University of Michigan, Ann Arbor, MI 48109, U.S.A.
}

\begin{abstract}
SUMMARY
A bonded joint finite element (FE) for a symmetric double lap joint is developed that is capable of predicting field quantities in the lap region. The element is a hybrid method and incorporates features of classical analytical and numerical methods. The element stiffness and load vector formulations have unique, load dependent, non-linear shape functions based on an analytical solution. The adaptive shape functions are formulated in terms of the dimensionless mechanical load fraction $\left(\overline{\bar{\phi}}_{P}\right)$ and total load $\left(\overline{\bar{\phi}}_{\text {tot }}\right)$ and are capable of predicting the thermal and mechanical load response. The bonded joint element has been implemented as a user element in the Abaqus ${ }^{\circledR}$ commercial FE code. A comparison of the stress predictions for the bonded joint element and a conventional 2D FE model is presented and are found to be in good agreement. Therefore, the element provides a computationally efficient and mesh-independent stress prediction. The single element reproduces the analytical solution with minimal analyst input and can be easily incorporated into early design and sizing studies. Copyright (C) 2009 John Wiley \& Sons, Ltd.
\end{abstract}

Received 11 June 2008; Revised 10 November 2008; Accepted 22 December 2008

KEY WORDS: composites; lap joint; temperature; orthotropic; embedded solution; single finite element

\section{INTRODUCTION}

Despite decades of development, the design and modeling of bonded joints is an active area of research. Conventional finite element (FE) models are the current state of the art and are widely available in the literature where work began as early as 1971 ([1,2] are early references). More

\footnotetext{
*Correspondence to: Anthony M. Waas, Department of Aerospace Engineering, University of Michigan, Ann Arbor, MI 48109, U.S.A.

†E-mail: dcw@umich.edu

${ }^{\ddagger}$ Post-doctoral scholar.

${ }^{\S}$ Professor of Aerospace Engineering.

Contract/grant sponsor: Space Vehicle Technology Institute; contract/grant number: NCC3-989

Contract/grant sponsor: NASA

Contract/grant sponsor: DOD
} 
recently, promising advances in cohesive zone [3-9], discrete cohesive zone [10-12], fracture mechanics [13], probabilistic prediction [14, 15], virtual crack closure technique [16-22] and other adhesive region models [23-26] have greatly increased the predictive capability of FE techniques. Cohesive zone models have been incorporated into commercial software including Abaqus ${ }^{\circledR}$ [27] and Genoa ${ }_{\circledR}[28]$, as well as freely available research codes like Tahoe ${ }^{\circledR}$ [29]. Despite their availability, the listed techniques are expensive and require extensive user expertise. There are ongoing efforts to develop rapid analysis techniques [30-32], a key enabling technology for vehicle designers.

Though models built with the tools listed above can be accurate, they rely on the presence of a meshed joint. Conventional volumetric elements represent the adherends; the adhesive is represented by volumetric elements or a discrete traction law. There is substantial overhead in creating and analyzing joints using these and other conventional FE-based numerical methods. Mesh generation and manipulation is an obstacle for anything beyond academic geometries. Mesh density is also a consideration, since the computational time for basic joints can be significant if non-linear material properties and material degradation criterion are included. As a result, there are ongoing efforts to evaluate analytical techniques that are less mesh dependent. For example, the composites affordability initiative has recommended a $p$-based analysis code for analysis of adhesively bonded joints, ${ }^{\mathbb{1}}$ since the use of $p$-based codes should be less mesh dependent than $h$-based FE codes. Similarly, Bednarcyk et al. [33] used a higher-order, semi-analytical theory (developed for functionally graded materials) to analyze a double lap and a bonded doubler joint. These techniques were reported to be less mesh dependent than $h$-based analysis methodologies. In this article, a bonded joint finite element (BJFE) is developed as a specialized element and technique for efficient joint analysis. The BJFE has no mesh dependency and requires minimal meshing overhead.

\section{BACKGROUND}

The objective of this article is to develop an element capable of predicting basic joint performance with a limited number of degrees of freedom (DOF) and with little meshing overhead. As a result, this element could be adopted for initial joint sizing in FE models at all system levels. The element is formulated to predict stress and strain fields of orthotropic constituents in thermal (or any scalar) and mechanical loading environments. The orthotropy of a joint is of particular concern in laminated composite materials since transverse properties are often significantly lower than in-plane properties in a laminate [34]. Temperature dependence is included since anisotropic materials (such a long fiber-reinforced composites) often require high-temperature curing cycles.

There are many factors that affect the stress field and associated bonded joint failure. These include adhesive spew [2] and the geometric discontinuity and unbounded stresses associated with stepwise geometries [35]. Additionally, material non-linearity has a significant effect on the stress field $[34,36]$ and requires a level of material characterization that is often unavailable early in an analysis cycle. All of the specialized joint analysis techniques (cohesive elements, the virtual crack closure technique and others) require material properties that can be difficult to obtain (such as critical energy release rates and cohesive strengths). In many circumstances, a designer has

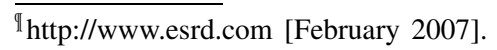




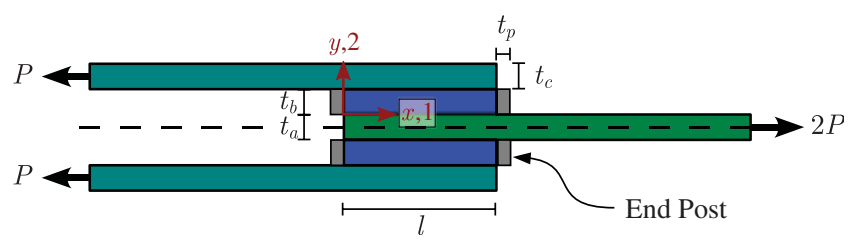

Figure 1. Schematic of the double lap joint with end posts.

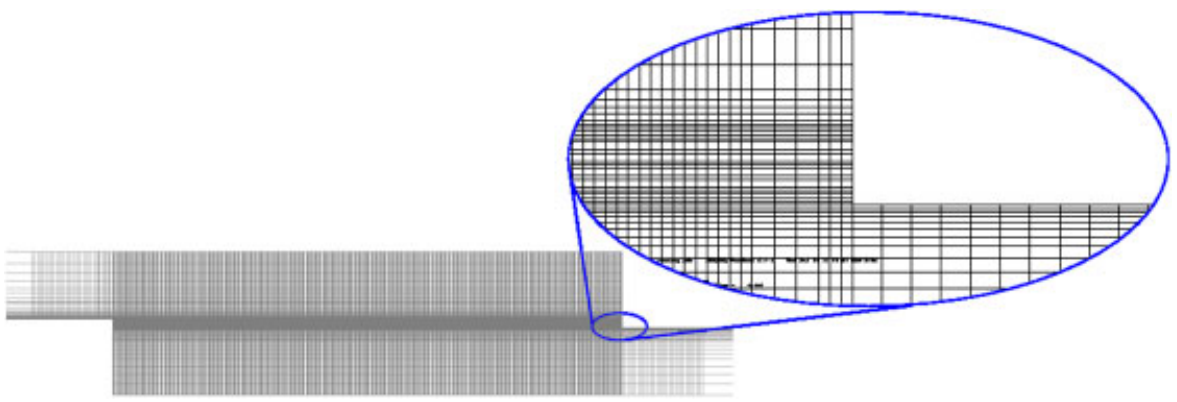

Figure 2. The FE mesh.

insufficient information or time to obtain a highly accurate solution and instead would prefer a simple analysis that captures primary effects. These types of analyses are often useful in trade studies and to identify likely problem areas needing further study.

With that goal in mind, it might be considered adequate to perform a conventional linear elastic FE analysis on an idealized geometry such as a joint with square corners (i.e. the double lap joint in Figures 1 and 2). In that solution type, however, the singular stress field causes a broad range of predicted stresses near the edges, particularly at the material interfaces. This is an undesirable and an unavoidable feature that emerges when a linear elastic material description is used for a corner consisting of two materials that are perfectly bonded. The utility of the linear elastic FE analysis is, therefore, somewhat limited with respect to joint to joint comparison.

For example, a double lap joint is schematically represented in Figure 1. The central adherend is referred to as material $a$ and the outer adherend is referred to as material $c$. Material $b$ is the adhesive and is thin in comparison to the adherends. A typical prediction of peel stress $\left(\bar{\sigma}_{b 22}\right)$ is illustrated in Figure 3." It is apparent that the peel stress can be determined as a function of longitudinal position over most of the joint. In the critical areas near the edges of the joint, however, the predicted stress varies widely and is mesh dependent. The severity of the mesh dependency is illustrated in Figure 4, where the predicted stress increases without bound with increasing element density. Even when non-linear material properties are assumed, which sometimes can ensure that the stress remains bounded [34], mesh dependency and convergence remain a concern. When this is the case, it is a common practice to create several costly meshes at different densities in order

\footnotetext{
"The typical result is taken from a model associated with Figures 1 and 2.
} 


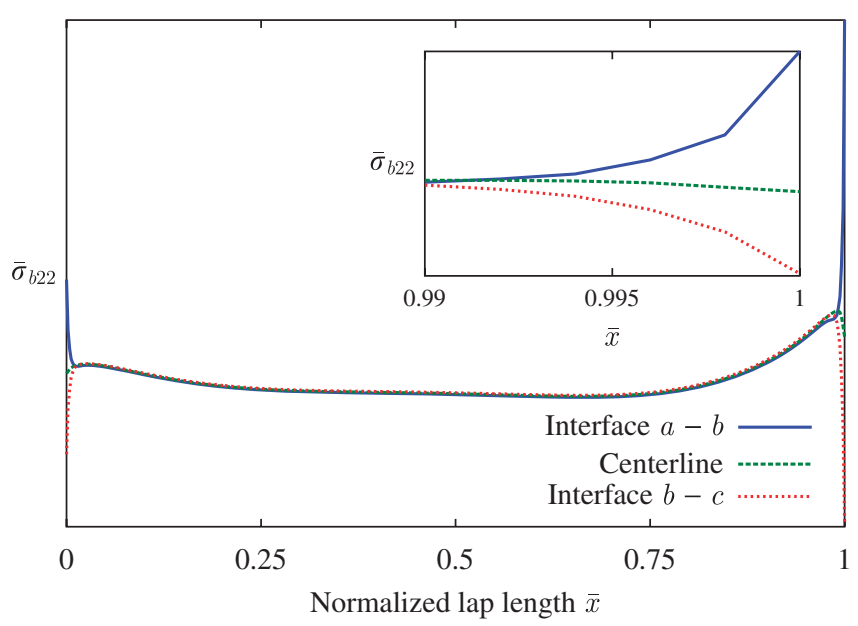

Figure 3. Typical linear elastic peel stress distribution due to mixed loading.

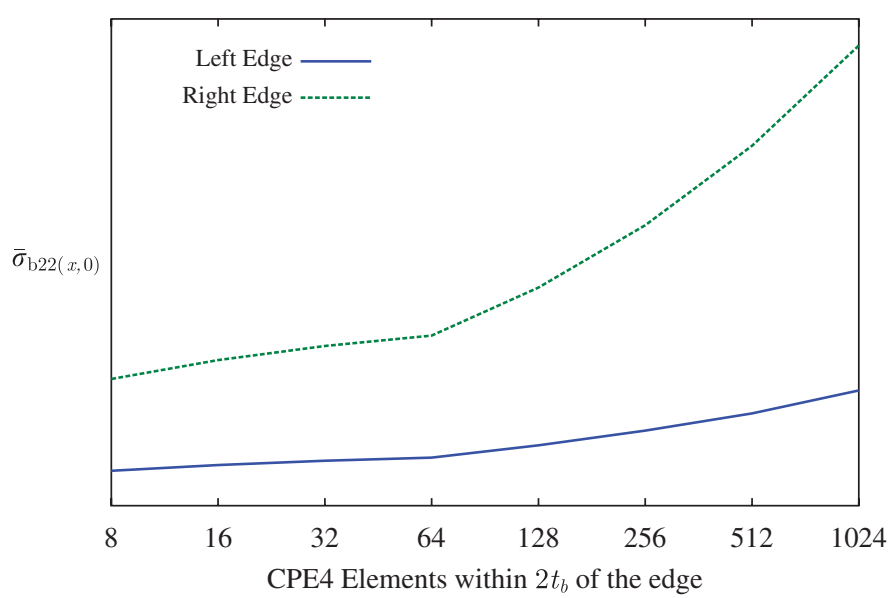

Figure 4. Typical mesh 'convergence' study for linear elastic stepwise geometry.

to verify that the stress results have converged. Smeltzer and Lundgren [32] is a recent example of this practice.

In view of the alternatives presented above and in order to be useful to an analyst, the BJFE must accurately represent the value of the most critical stresses in the joint while consistently and correctly predicting the trends from joint to joint. It must accomplish this with no mesh dependency and insignificant meshing overhead. Further, its use must not directly burden the user with the significant calculations typically associated with analytical solutions such as those in [37]. In the remaining sections of this article, a bonded joint element is developed to meet these requirements. Planned extensions to the BJFE are expected to capture the primary effects of failure mechanisms when a sufficient material description is available early in the design cycle. 


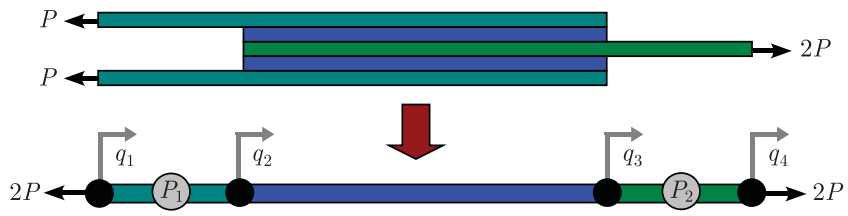

Figure 5. Symmetric double lap joint and BJFE representation.

The predicted stresses and resulting displacements have non-trivial spatial non-linearities and are not well represented by a small number of linear or quadratic FEs. The displacement field, however, can be represented by appropriate load-dependent adaptive shape functions $N\left(\overline{\bar{\phi}}_{P}\right)$ with a single element. Based on the stress predictions presented in Section 3, an element with a load-dependent stiffness matrix $K\left(\overline{\bar{\phi}}_{P}\right)$ and consistent loading vector $F\left(\overline{\bar{\phi}}_{P}\right)$ are presented in the remaining sections.

\subsection{The element concept}

Figure 5 is a schematic of the BJFE element concept. In it, the complete lap joint is replaced with a single FE with 6 DOF. Four displacement DOF $\left(q_{1}, q_{2}, q_{3}, q_{4}\right)$ are used to represent four discrete displacement locations and two internal DOF $\left(P_{1}, P_{2}\right)$ are used to determine the load 'character'. The displacement field is interpolated with application-specific adaptive shape functions, detailed in Section 4. The load 'character' is a ratio of the thermal and longitudinal mechanical loads and governs the internal displacement field via the adaptive shape functions [37]. The adaptive shape functions allow accurate predictions of the stress and strain field in a double lap joint through use of a single FE.

\section{DERIVATION OF THE ADVANCED SHEAR AND PEEL MODEL}

In [37], two dimensionless solutions are developed for a symmetric, orthotropic double lap joint subjected to thermomechanical loading. The primary purpose of those solutions is to establish relevant dimensionless parameters that predict the stress field. Using those parameters and the 'simple' analytical solutions that they are based on, the effects of various material and loading properties on a joint stress field can be determined. The solutions were not precise in their predictions, despite being adequate to correctly predict trends. It is anticipated that the user of the BJFE would desire more precision; therefore, a more accurate analytical solution is developed in this section for the BJFE.

The double lap joint's response to thermal and longitudinal mechanical load is modeled. The material is assumed to be linear elastic and orthotropic with linear orthotropic thermal expansion. The joint is assumed to deform in plane strain.** The solution is more 'complex' than those that were presented in [37]. It is developed, however, for automated use within the BJFE where solution complexity is no longer an issue. The adaptive shape functions derived in Section 4 are based on the equilibrium stresses developed in this section.

\footnotetext{
${ }^{* *}$ The material constitutive response is given by (A1).
} 


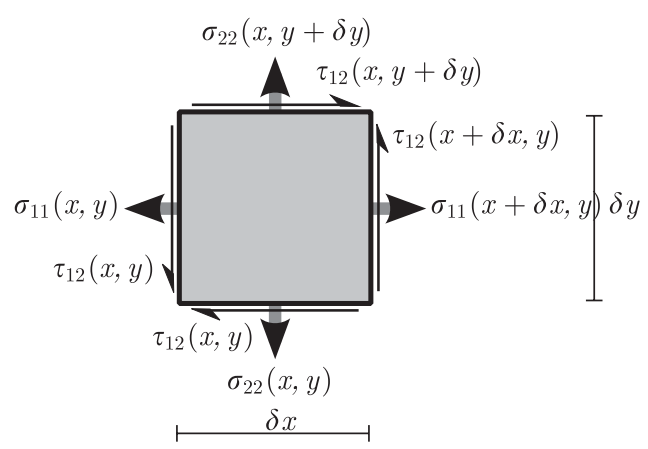

Figure 6. Generalized equilibrium parallelepiped.

Figure 6 shows a general 2D parallelepiped. Force equilibrium in 1 and 2 directions can be written as:

$$
\begin{aligned}
\sum F_{1} & =0 \\
& =\delta y\left(\sigma_{11}(x+\delta x, y)-\sigma_{11}(x, y)\right)+\delta x\left(\tau_{12}(x, y+\delta y)-\tau_{12}(x, y)\right) \\
\sum F_{2} & =0 \\
& =\delta x\left(\sigma_{22}(x, y+\delta y)-\sigma_{22}(x, y)\right)+\delta y\left(\tau_{12}(x+\delta x, y)-\tau_{12}(x, y)\right)
\end{aligned}
$$

Equation (1) can be rewritten as the shear-normal stress relationship for each constituent:

$$
\begin{aligned}
& \frac{\partial \sigma_{11}(x, y)}{\partial x}=-\frac{\partial \tau_{12}(x, y)}{\partial y} \\
& \frac{\partial \sigma_{22}(x, y)}{\partial y}=-\frac{\partial \tau_{12}(x, y)}{\partial x}
\end{aligned}
$$

In [37], the adherends were assumed to carry only longitudinal normal stress and the adhesive was assumed to carry only shear and peel stresses. In this article, those assumptions are relaxed so that the adherends also transmit shear stress. For convenience, the adherend shear stress fields are assumed to vary linearly in $y$ throughout the specimen. ${ }^{\dagger \dagger}$ As a result, (2) dictates that the adherend longitudinal normal stresses are functions of $x$ only; the peel stresses are linear functions of $x$ and $y$. The longitudinal normal stress in the adhesive is still assumed to be zero, therefore (2) dictates that the shear stress in the adhesive is a function of $x$ only. ${ }^{\ddagger \ddagger}$

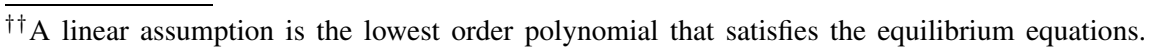

¥The assumption of zero longitudinal normal stress in the adhesive greatly simplifies the calculations and is reasonable for calculating stress as long as the strain energy due to this stress component is small relative to the total strain energy. The model breaks down in joints with similar adherend thermal expansion and a large differential thermal expansion relative to the adhesive. In those joints, the longitudinal thermal stress of the adhesive will be greater than the adhesive shear and peel stresses. Therefore, a different type of analysis is appropriate.
} 
Traction-free boundaries are present on the top and the bottom surfaces of the joint. The centerline of the central adherend is free of shear due to symmetry. These requirements are expressed as:

$$
\begin{aligned}
& \tau_{c 12}\left(x, t_{b}+t_{c}\right)=0 \\
& \sigma_{c 22}\left(x, t_{b}+t_{c}\right)=0 \\
& \tau_{a 12}\left(x,-\frac{t_{a}}{2}\right)=0
\end{aligned}
$$

Stress continuity at the joint interfaces requires:

$$
\begin{aligned}
\sigma_{b 22}(x, 0) & =\sigma_{a 22}(x, 0) \\
\sigma_{c 22}\left(x, t_{b}\right) & =\sigma_{b 22}\left(x, t_{b}\right) \\
\tau_{b 12}(x, 0) & =\tau_{a 12}(x, 0) \\
\tau_{c 12}\left(x, t_{b}\right) & =\tau_{b 12}\left(x, t_{b}\right)
\end{aligned}
$$

Finally, longitudinal normal stress boundary conditions are imposed by the mechanical loads at the edges of central adherend $a$ and are expressed as:

$$
\begin{aligned}
\sigma_{a 11}(0) & =0 \\
\sigma_{a 11}(l) & =\frac{2 P}{t_{a}}
\end{aligned}
$$

By sequentially writing a linear form for each stress component (using the stress field assumptions) and by applying boundary and continuity conditions to determine the linear constants, equations can be written for each stress component in terms of the central adherend stress $\sigma_{a 11}(x)$. The process is as described in [37] with the addition of several stress components $\left(\tau_{a 12}(x, y)\right.$, $\left.\sigma_{a 22}(x, y), \tau_{c 12}(x, y), \sigma_{c 22}(x, y)\right)$. The resulting stress equations are detailed on the left side of Table I.

In addition to the boundary conditions specified in (3)-(5), the adhesive edge shear stress is forced to zero using the 'end post' technique described in [37]. The technique, an extension of [38], ensures that the traction-free boundary condition at the adhesive free edges is captured. An infinitesimal end post transfers the edge shear stress to the adherend. The stresses in the end posts are also listed on the left side of Table I.

The solution for the central adherend normal stress $\left(\sigma_{a 11}(x)\right)$ is computed via application of the principle of virtual forces as detailed in Appendix B. In a brief summary of the computation, each stress component is a function of $\sigma_{a 11}(x)$. For each component, a corresponding virtual stress component is written in terms of the virtual normal stress $\hat{\sigma}_{a 11}(x)$. (The virtual stress components are listed on the right side of Table I.) By integrating potential energy over the volume of the joint and minimizing for any admissible $\hat{\sigma}_{a 11}(x)$, a differential equation is written for the central adherend stress field $\sigma_{a 11}(x)$ as a function of all material properties and loads

$$
\frac{\mathrm{d}^{4} \sigma_{a 11}(x)}{\mathrm{d} x^{4}}+\beta \frac{\mathrm{d}^{2} \sigma_{a 11}(x)}{\mathrm{d} x^{2}}+\gamma \sigma_{a 11}(x)+\phi_{\Delta T}+\phi_{P}=0
$$


Table I. Stresses and virtual stresses in the BJFE solution.

\begin{tabular}{ll}
\hline Equilibrium normal stress & \multicolumn{1}{c}{ Virtual normal stress } \\
\hline$\sigma_{a 11}(x)$ & $\hat{\sigma}_{a 11}(x)$ \\
$\sigma_{c 11}(x)=\frac{P}{t_{c}}-\frac{t_{a} \sigma_{a 11}(x)}{2 t_{c}}$ & $\hat{\sigma}_{c 11}(x, y)=-\frac{t_{a} \hat{\sigma}_{a 11}(x)}{2 t_{c}}$ \\
$\sigma_{a 22}(x, y)=\frac{\mathrm{d}^{2}}{\mathrm{~d} x^{2}} \sigma_{a 11}(x)\left(\frac{y^{2}+t_{a} y}{2}-\frac{t_{a}\left(t_{c}+2 t_{b}\right)}{4}\right)$ & $\hat{\sigma}_{a 22}(x, y)=\frac{\mathrm{d}^{2}}{\mathrm{~d} x^{2}} \hat{\sigma}_{a 11}(x)\left(\frac{y^{2}+t_{a} y}{2}-\frac{t_{a}\left(t_{c}+2 t_{b}\right)}{4}\right)$ \\
$\sigma_{b 22}(x, y)=\frac{t_{a}\left(\frac{\mathrm{d}^{2}}{\mathrm{~d} x^{2}} \sigma_{a 11}(x)\right)\left(2 y-t_{c}-2 t_{b}\right)}{4}$ & $\hat{\sigma}_{b 22}(x, y)=\frac{t_{a}\left(\frac{\mathrm{d}^{2}}{\mathrm{~d} x^{2}} \hat{\sigma}_{a 11}(x)\right)\left(2 y-t_{c}-2 t_{b}\right)}{4}$ \\
$\sigma_{c 22}(x, y)=-\frac{t_{a}\left(\frac{\mathrm{d}^{2}}{\mathrm{~d} x^{2}} \sigma_{a 11}(x)\right)\left(y-t_{c}-t_{b}\right)^{2}}{4 t_{c}}$ & $\hat{\sigma}_{c 22}(x, y)=-\frac{t_{a}\left(\frac{\mathrm{d}^{2}}{\mathrm{~d} x^{2}} \hat{\sigma}_{a 11}(x)\right)\left(y-t_{c}-t_{b}\right)^{2}}{4 t_{c}}$ \\
Equilibrium shear stress & Virtual shear stress \\
$\tau_{a 12}(x, y)=-\frac{\frac{\mathrm{d}}{\mathrm{d} x} \sigma_{a 11}(x)\left(2 y+t_{a}\right)}{2}$ & $\hat{\tau}_{a 12}(x, y)=-\frac{\frac{\mathrm{d}}{\mathrm{d}} \hat{\sigma}_{a 11}(x)\left(2 y+t_{a}\right)}{2}$ \\
$\tau_{b 12}(x)=-\frac{t_{a}\left(\frac{\mathrm{d}}{\mathrm{d} x} \sigma_{a 11}(x)\right)}{2}$ & $\hat{\tau}_{b 12}(x, y)=-\frac{t_{a}\left(\frac{\mathrm{d}}{\mathrm{d} x} \hat{\sigma}_{a 11}(x)\right)}{2}$ \\
$\tau_{c 12}(x, y)=\frac{t_{a}\left(\frac{\mathrm{d}}{\mathrm{d} x} \sigma_{a 11}(x)\right)\left(y-t_{c}-t_{b}\right)}{2 t_{c}}$ & $\hat{\tau}_{c 12}(x, y)=\frac{t_{a}\left(\frac{\mathrm{d}}{\mathrm{d} x} \hat{\sigma}_{a 11}(x)\right)\left(y-t_{c}-t_{b}\right)}{2 t_{c}}$ \\
Equilibrium end post stress & $\operatorname{Virtual}_{c}$ end post stress \\
$\sigma_{p 22}(\bar{x}=0, y)=\frac{t_{a}\left(\frac{\mathrm{d}}{\mathrm{d} x} \sigma_{a 11}(x)\right)\left(y-t_{b}\right)}{2 t_{p}}$ & $\hat{\sigma}_{p 22}(\bar{x}=0, y)=\frac{t_{a}\left(\frac{\mathrm{d}}{\mathrm{d} x} \hat{\sigma}_{a 11}(x)\right)\left(y-t_{b}\right)}{2 t_{p}}$ \\
$\sigma_{p 22}(\bar{x}=1, y)=-\frac{t_{a}\left(\frac{\mathrm{d}}{\mathrm{d} x} \sigma_{a 11}(x)\right)\left(y-t_{b}\right)}{2 t_{p}}$ & $\hat{\sigma}_{p 22}(\bar{x}=1, y)=-\frac{t_{a}\left(\frac{\mathrm{d}}{\mathrm{d} x} \hat{\sigma}_{a 11}(x)\right)\left(y-t_{b}\right)}{2 t_{p}}$ \\
\hline
\end{tabular}

In (6), all material terms have been grouped according to their order of derivative $(\beta$ and $\gamma)$ and all load terms have been grouped into thermal $\left(\phi_{\Delta T}\right)$ and mechanical parameters $\left(\phi_{P}\right)$. Equation (6) can be non-dimensionalized with the following substitutions:

$$
\begin{aligned}
\bar{x} & =\frac{x}{l} \\
\bar{\beta} & =l^{2} \beta \\
\bar{\gamma} & =l^{4} \gamma \\
\bar{\phi}_{\Delta T} & =\frac{l^{4}}{E_{a 11}} \phi_{\Delta T} \\
\bar{\phi}_{P} & =\frac{l^{4}}{E_{a 11}} \phi_{P} \\
\overline{\bar{\phi}}_{\text {tot }} & =\bar{\phi}_{P}+\bar{\phi}_{\Delta T} \\
\overline{\bar{\phi}}_{P} & =\frac{\bar{\phi}_{P}}{\overline{\bar{\phi}}_{\mathrm{tot}}} \\
\overline{\bar{\sigma}}_{\kappa i j}(\bar{x}) & =\frac{\sigma_{\kappa i j}(l \bar{x})}{E_{a 11} \overline{\bar{\phi}}_{\mathrm{tot}}}
\end{aligned}
$$


In (7), $\bar{x}$ is the dimensionless spatial coordinate measured from the left edge of the joint, $\bar{\beta}$ and $\bar{\gamma}$ are dimensionless material parameters, and $\bar{\phi}_{P}$ and $\bar{\phi}_{\Delta T}$ are the dimensionless mechanical and thermal loads. The dimensionless total load is $\overline{\bar{\phi}}_{\text {tot }}$ and is used to further normalize the stresses $\left(\overline{\bar{\sigma}}_{\kappa i j}(\bar{x})\right)$. Similarly, the mechanical fraction of the dimensionless total load is $\overline{\bar{\phi}}_{P}$. Each of the terms in (7) are explicitly reported in terms of the constitutive and load quantities in Appendix D. After substitution, (6) becomes:

$$
\frac{\partial^{4}}{\partial \bar{x}^{4}} \overline{\bar{\sigma}}_{a 11}\left(\bar{x}, \overline{\bar{\phi}}_{P}\right)+\bar{\beta} \frac{\partial^{2}}{\partial \bar{x}^{2}} \overline{\bar{\sigma}}_{a 11}\left(\bar{x}, \overline{\bar{\phi}}_{P}\right)+\bar{\gamma}_{\bar{\sigma}} \bar{\sigma}_{a 11}\left(\bar{x}, \overline{\bar{\phi}}_{P}\right)+1=0
$$

A solution is:

$$
\overline{\bar{\sigma}}_{a 11}\left(\bar{x}, \overline{\bar{\phi}}_{P}\right)=\overline{\bar{A}}\left(\overline{\bar{\phi}}_{P}\right) \mathrm{e}^{\bar{\lambda}_{1} \bar{x}}+\overline{\bar{B}}\left(\overline{\bar{\phi}}_{P}\right) \mathrm{e}^{-\bar{\lambda}_{1} \bar{x}}+\overline{\bar{C}}\left(\overline{\bar{\phi}}_{P}\right) \mathrm{e}^{\bar{\lambda}_{3} \bar{x}}+\overline{\bar{D}}\left(\overline{\bar{\phi}}_{P}\right) \mathrm{e}^{-\bar{\lambda}_{3} \bar{x}}-\frac{1}{\bar{\gamma}}
$$

This solution to (6) is an equation for the normalized central adherend stress $\left(\overline{\bar{\sigma}}_{a 11}\left(\bar{x}, \overline{\bar{\phi}}_{P}\right)\right)$, from which all stress components can be determined using (7) and the equations in Table I. As in [37], the material parameters in (9) $\left(\bar{\lambda}_{1}\right.$ and $\left.\bar{\lambda}_{3}\right)$ are recast as the roots of the bi-quadratic differential equation:

$$
\bar{\lambda}^{2}=\frac{-\bar{\beta} \pm \sqrt{\bar{\beta}^{2}-4 \bar{\gamma}}}{2}
$$

The equations for the dimensionless basis functions $(\overline{\bar{A}}, \overline{\bar{B}}, \overline{\bar{C}}, \overline{\bar{D}})$ are determined by application of the boundary conditions ${ }^{\S}$ :

$$
\begin{aligned}
& \overline{\bar{A}}\left(\overline{\bar{\phi}}_{P}\right)=\frac{\mu_{3} \mu_{A_{P}}}{\mu_{1} \mu_{2}} \overline{\bar{\phi}}_{P}+\frac{\mu_{A_{\Delta T}}}{\mu_{1}} \\
& \overline{\bar{B}}\left(\overline{\bar{\phi}}_{P}\right)=\frac{\mu_{3} \mu_{B_{P}}}{\mu_{1} \mu_{2}} \overline{\bar{\phi}}_{P}+\frac{\mu_{B_{\Delta T}}}{\mu_{1}} \\
& \overline{\bar{C}}\left(\overline{\bar{\phi}}_{P}\right)=\frac{\mu_{3} \mu_{C_{P}}}{\mu_{1} \mu_{2}} \overline{\bar{\phi}}_{P}+\frac{\mu_{C_{\Delta T}}}{\mu_{1}} \\
& \overline{\bar{D}}\left(\overline{\bar{\phi}}_{P}\right)=\frac{\mu_{3} \mu_{D_{P}}}{\mu_{1} \mu_{2}} \overline{\bar{\phi}}_{P}+\frac{\mu_{D_{\Delta T}}}{\mu_{1}}
\end{aligned}
$$

In (11), the basis functions are linear in the mechanical fraction of the total load $\left(\overline{\bar{\phi}}_{P}\right)$. As a result, they effectively separate the thermal and mechanical loads. The basis functions are composed of several material parameter combinations, denoted by $\mu$, whose values are listed in Appendix D.8. In combination, (9)-(11) provide a solution to the double lap joint that is sufficiently accurate to accomplish the goals of the BJFE.

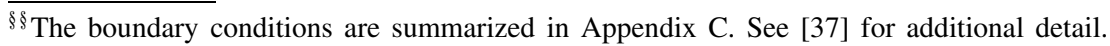




\section{FORMULATION OF THE FE}

Figure 5 is a schematic of the BJFE. The element is one dimensional; all displacement DOF are oriented along the 1-axis. Two of the displacement DOF ( $q_{1}$ and $\left.q_{4}\right)$ are external and connect the joint element to the external structure. The remaining displacement DOF are internal to the element and are used in conjunction with supplemental equations in order to determine the mechanical

loading fraction $\left(\overline{\bar{\phi}}_{P}\right)$ required by the adaptive shape function. The mechanical load that is carried across the joint can be calculated using internal DOF $P_{1}$ and $P_{2}$.

The derivation of the element is presented in stages. First, the outer section sub-elements are formulated from the equilibrium stress equation. The formulation is subsequently generalized for the lap region sub-element. An equilibrium formulation is required since the displacement field is governed by an adaptive shape function that is dependent on load character.

\subsection{Stiffness and load contribution of the adherends outside of the lap region}

The stress in the adherend structures outside the lap region is assumed to have no transverse stress $\left(\sigma_{k 22}=0\right)$. The orthotropic adherend constitutive relationship is given in (A1). All Poisson terms will be set to zero in the initial portion of the derivation, thus, the sub-elements outside the lap region are equivalent to truss elements. A more general analysis would include these Poisson terms, however, retaining them in this derivation deters the demonstration without adding value.

As a preface to the remainder of this section, the following derivation may seem unnecessary since the truss element has linear shape functions and is well understood. The reader could skip to the next section without loss of substance. The subsequent derivation of the lap region's adaptive shape functions, however, is completed using the same steps. The intermediate results of that derivation are too long to be included in the text. As a result, a detailed derivation is presented for this sub-element where it can be easily understood.

With a view to deriving the adaptive shape functions in the lap region, the stress field in the outer center adherend is written directly from equilibrium

$$
\sigma_{a 11}(\bar{x})=\frac{P}{t_{a}}
$$

where $\bar{x}$ is the natural coordinate of this section, defined as:

$$
\bar{x}=\frac{x}{l_{e}}
$$

The sub-element local $(x, y)$ directions are defined from the left edge of the sub-element and the sub-element length is $l_{e}$ as illustrated in Figure 7(a). The sub-element displacement DOF are temporarily replaced by a single extensional DOF given by:

$$
q_{e}=q_{4}-q_{3}
$$

From (A1) and (12), the strain field can be written as:

$$
\varepsilon_{a 11}(\bar{x})=\frac{\sigma_{a 11}(\bar{x})}{E_{a 11}}+\alpha_{a 11} \Delta T
$$




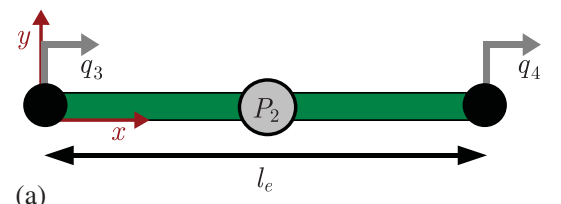

(a)

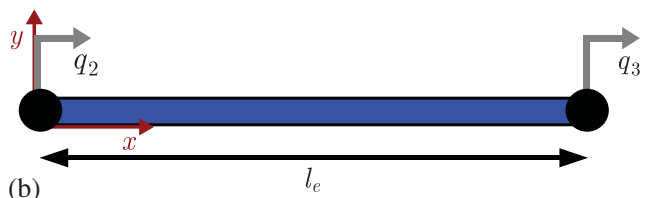

Figure 7. BJFE sub-elements: (a) sub-element for the central adherend outside of the lap region and (b) sub-element for the lap region of the double lap joint.

Integration of the strain field yields the longitudinal displacement field

$$
u_{a}(\bar{x})=\int_{0}^{\bar{x}} \varepsilon_{a 11}(\bar{x})\left(\frac{\mathrm{d} x}{\mathrm{~d} \bar{x}}\right) \mathrm{d} \bar{x}+u_{\mathrm{rb}}
$$

where $u_{\mathrm{rb}}$ is an integration constant that represents rigid body displacement of the element. The extension $\left(q_{e}\right)$ is given by:

$$
\begin{aligned}
q_{e} & =u_{a}(\bar{x}=1)-u_{\mathrm{rb}} \\
& =l_{e}\left[\frac{P}{E_{a 11} t_{a}}+\alpha_{a 11} \Delta T\right]
\end{aligned}
$$

The intent of this section is to understand the subsequent lap region derivation, therefore, recall that the joint section stress field given in (9) is written in terms of dimensionless loads. To generalize the loads for this sub-element, non-dimensionalizing substitutions are made:

$$
\begin{aligned}
P & =\bar{\psi}_{P} E_{a 11} t_{a} \\
\Delta T & =\frac{\bar{\psi}_{T}}{\alpha_{a 11}}
\end{aligned}
$$

Parameters $\bar{\psi}_{\Delta T}$ and $\bar{\psi}_{P}$ are dimensionless thermal and mechanical loads. Additionally, all critical values can be written in terms of the mechanical load fraction $\left(\overline{\bar{\psi}}_{P}\right)$ and the total load $\left(\overline{\bar{\psi}}_{\text {tot }}\right)$ :

$$
\begin{aligned}
& \overline{\bar{\psi}}_{\text {tot }}=\bar{\psi}_{T}+\bar{\psi}_{P} \\
& \bar{\psi}_{P}=\overline{\bar{\psi}}_{\text {tot }} \overline{\bar{\psi}}_{P} \\
& \bar{\psi}_{T}=\overline{\bar{\psi}}_{\text {tot }}\left(1-\overline{\bar{\psi}}_{P}\right)
\end{aligned}
$$

Combining Equations (12), (15)-(17), and (19), the longitudinal displacement field can be written as

$$
u_{a}(\bar{x})=l_{e} \overline{\bar{\psi}}_{\mathrm{tot}} \bar{x}
$$


and the extension DOF can be written as:

$$
q_{e}=l_{e} \overline{\bar{\psi}}_{\mathrm{tot}}
$$

The displacement field of (20) is written in terms of the unknown total load $\left(\overline{\bar{\psi}}_{\text {tot }}\right)$. Using (21), the total load can be isolated as a linear function of the extension DOF $\left(q_{e}\right)$,

$$
\overline{\bar{\psi}}_{\mathrm{tot}}=\frac{q_{e}}{l_{e}}
$$

In (20) and (22), a linear displacement field is recovered and can be written as a shape function $N\left(\bar{x}, \overline{\bar{\psi}}{ }_{P}\right)$

$$
\begin{aligned}
u_{a}(\bar{x}) & =\bar{x} q_{e} \\
& =N\left(\bar{x}, \overline{\bar{\psi}}_{P}\right) q_{e}
\end{aligned}
$$

As the shape function has been determined from equilibrium, the mechanical load fraction $\left(\overline{\bar{\psi}}_{P}\right)$ is implicitly included in (22) and (23) (although it has been eliminated). Equation (23) is otherwise unremarkable, however, it is a necessary step in the process of deriving a shape function from the equilibrium equations. ${ }^{\text {III }}$ Using (23), the strain and stress can be written in terms of $q_{e}$ and the shape function derivative $B\left(\bar{x}, \overline{\bar{\psi}}_{P}\right)$,

$$
\begin{aligned}
\varepsilon_{a 11}(\bar{x}) & =\left(\frac{\mathrm{d} \bar{x}}{\mathrm{~d} x}\right) \frac{\mathrm{d}}{\mathrm{d} \bar{x}} u_{a}(\bar{x}) \\
& =\frac{B\left(\bar{x}, \overline{\bar{\psi}}_{P}\right) q_{e}}{l_{e}} \\
\sigma_{a 11}(\bar{x}) & =E_{a 11}\left(\varepsilon_{a 11}-\alpha_{a 11} \Delta T\right) \\
& =E_{a 11}\left(\frac{B\left(\bar{x}, \overline{\bar{\psi}}_{P}\right) q_{e}}{l_{e}}-\alpha_{a 11} \Delta T\right)
\end{aligned}
$$

In the example of the central adherend outside the lap section, $B\left(\bar{x}, \overline{\bar{\psi}}_{P}\right)=1$.

The strain energy and external work terms are:

$$
\begin{aligned}
U & =\int_{0}^{t_{a}} \int_{0}^{1} \sigma_{a 11}\left(\bar{x}, \overline{\bar{\psi}}_{P}\right) \delta \varepsilon_{a 11}\left(\bar{x}, \overline{\bar{\psi}}_{P}\right)\left(\frac{\mathrm{d} x}{\mathrm{~d} \bar{x}}\right) \mathrm{d} \bar{x} \mathrm{~d} y \\
W & =P q_{e}
\end{aligned}
$$

In contrast to (12), which was used to obtain the shape functions, (25) and (26) are written in terms of the temperature change and load $(\Delta T$ and $P)$. This is necessary to correctly compute the strain energy and work required for obtaining the stiffness matrix and load vector.

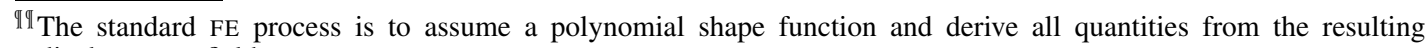
displacement field.
} 
Restoring the discrete displacements $\left(q_{3}, q_{4}\right)$ in place of the extension $\left(q_{e}\right)$, the strain energy is:

$$
U=\frac{E_{a 11}\left(q_{4}-q_{3}\right) \int_{y_{0}}^{y_{1}} \int_{0}^{1} B\left(\bar{x}, \overline{\bar{\psi}}_{P}\right)\left(\left(q_{4}-q_{3}\right) B\left(\bar{x}, \overline{\bar{\psi}}_{P}\right)-\alpha_{a 11} l_{e} \Delta T\right) \mathrm{d} \bar{x} \mathrm{~d} y}{2 l_{e}}
$$

With the work and strain energy fully defined, the potential energy equation can be used to extract the stiffness matrix and load vector:

$$
\begin{aligned}
\frac{\mathrm{d}}{\mathrm{d} \mathbf{q}_{e}} \Pi & =\frac{\mathrm{d}}{\mathrm{d} \mathbf{q}_{e}}(U-W) \\
& =0 \\
& \rightarrow K_{e} \mathbf{q}_{e}=\mathbf{F}_{e}
\end{aligned}
$$

The sub-element stiffness matrix, $K_{e}$, is

$$
K_{e}=\frac{\sum_{\kappa} E_{\kappa 11} \int_{y_{\kappa} 0}^{y_{\kappa 1}} \int_{0}^{1} B_{\kappa}^{2}\left(\bar{x}, \overline{\bar{\psi}}_{P}\right) \mathrm{d} \bar{x} \mathrm{~d} y_{\kappa}}{l_{e}}\left[\begin{array}{cc}
1 & -1 \\
-1 & 1
\end{array}\right]
$$

and the sub-element load vector, $\mathbf{F}$, is:

$$
\mathbf{F}_{e}=P\left\{\begin{array}{c}
-1 \\
1
\end{array}\right\}+\sum_{\kappa} \alpha_{\kappa 11} E_{\kappa 11}\left(\int_{y_{0}}^{y_{1}} \int_{0}^{1} B_{\kappa}\left(\bar{x}, \overline{\bar{\psi}}_{P}\right) \mathrm{d} \bar{x} \mathrm{~d} y_{\kappa}\right) \Delta T\left\{\begin{array}{c}
-1 \\
1
\end{array}\right\}
$$

In this section which focuses on the central adherend sub-element, the summations in (29) and (30) include only the central adherend $(\kappa=a)$. It is evident that when $B\left(\bar{x}, \overline{\bar{\psi}}_{P}\right)=1$ (for this subelement), the appropriate truss element stiffness is recovered. Therefore, the sub-element stiffness and load quantities can be derived from equilibrium using non-dimensionalized loads and their ratios. Identical sub-element formulations are used for the central adherend and outer adherends (external to the lap region).

4.1.1. Stiffness and load contribution of the adhesively lap section. In the prior section, a general method was developed for calculating a stiffness matrix and load vector which are load dependent. More specifically, the stiffness matrix and load vector were derived as functions of the ratio of dimensionless thermal and mechanical loads, rewritten in terms of the mechanical load fraction $\left(\overline{\bar{\psi}}_{P}\right)$. Although the truss-type element derivation necessarily resulted in a linear displacement field and a load independent stiffness matrix, the method is general. In this section, it is used to develop an adaptive shape function for the displacement field of the lap region of a symmetric double lap joint.

Following the order of the derivation in the prior section and applying it to the sub-element in Figure 7(b), the equilibrium stress field must be known. Within the assumptions of its 
derivation, "I| the double lap joint stress field has the following components for the adherends and adhesive:

$$
\begin{aligned}
\sigma_{a 11}\left(\bar{x}, \overline{\bar{\phi}}_{P}, \overline{\bar{\phi}}_{\text {tot }}\right) & =E_{a 11} \overline{\bar{\phi}}_{\text {tot }}\left(\overline{\bar{A}}\left(\overline{\bar{\phi}}_{P}\right) e^{\bar{\lambda}_{1} \bar{x}}+\overline{\bar{B}}\left(\overline{\bar{\phi}}_{P}\right) e^{-\bar{\lambda}_{1} \bar{x}}+\overline{\bar{C}}\left(\overline{\bar{\phi}}_{P}\right) e^{\bar{\lambda}_{3} \bar{x}}+\overline{\bar{D}}\left(\overline{\bar{\phi}}_{P}\right) e^{-\bar{\lambda}_{3} \bar{x}}-\frac{1}{\bar{\gamma}}\right) \\
\sigma_{b 12}\left(\bar{x}, \overline{\bar{\phi}}_{P}, \overline{\bar{\phi}}_{\text {tot }}\right) & =-\frac{t_{a}}{2}\left(\frac{\mathrm{d} \bar{x}}{\mathrm{~d} x}\right)\left(\frac{\mathrm{d}}{\mathrm{d} \bar{x}} \sigma_{a 11}\left(\bar{x}, \overline{\bar{\phi}}_{P}, \overline{\bar{\phi}}_{\text {tot }}\right)\right) \\
\sigma_{b 22}\left(\bar{x}, y, \overline{\bar{\phi}}_{P}, \overline{\bar{\phi}}_{\text {tot }}\right) & =\frac{t_{a}}{2}\left(\frac{\mathrm{d} \bar{x}}{\mathrm{~d} x}\right)^{2}\left(\frac{\mathrm{d}^{2}}{\mathrm{~d} \bar{x}^{2}} \sigma_{a 11}\left(\bar{x}, \overline{\bar{\phi}}_{P}, \overline{\bar{\phi}}_{\text {tot }}\right)\right)\left(y-t_{b}\right) \\
\sigma_{c 11}\left(\bar{x}, \overline{\bar{\phi}}_{P}, \overline{\bar{\phi}}_{\text {tot }}\right) & =\frac{P}{t_{c}}-\frac{t_{a} \sigma_{a 11}\left(\bar{x}, \overline{\bar{\phi}}_{P}, \overline{\bar{\phi}}_{\text {tot }}\right)}{2 t_{c}}
\end{aligned}
$$

The stress components not listed in (31) can be determined but are of less interest.

In the discrete space of the FE model, the known (or desired) quantities are the applied temperature change ( $\Delta T$, assumed to be constant throughout the element) and the nodal loads and displacements. The load quantities must be recast into their dimensionless forms to conform to the governing equation for $\sigma_{a 11}(x)$. Non-dimensionalizing parameters are defined so that:

$$
\begin{gathered}
\Delta T=\frac{\Theta}{\theta_{\Delta T}} \bar{\phi}_{\Delta T} \\
P=\frac{\Theta}{\theta_{P}} \bar{\phi}_{P}
\end{gathered}
$$

Application of (32) to the equilibrium stress field and constitutive law, the strain can be written as a linear function of the total load $\overline{\bar{\phi}}_{\text {tot }}$ :

$$
\begin{aligned}
& \frac{\varepsilon_{a 11}\left(\bar{x}, \overline{\bar{\phi}}_{P}, \overline{\bar{\phi}}_{\text {tot }}\right)}{\overline{\bar{\phi}}_{\text {tot }}}=\left(1-v_{a 13} v_{a 31}\right) \\
& \cdot\left(\mathrm{e}^{-\bar{\lambda}_{3} \bar{x}} \overline{\bar{D}}\left(\overline{\bar{\phi}}_{P}\right)+\mathrm{e}^{\bar{\lambda}_{3} \bar{x}} \overline{\bar{C}}\left(\overline{\bar{\phi}}_{P}\right)+\mathrm{e}^{-\bar{\lambda}_{1} \bar{x}} \overline{\bar{B}}\left(\overline{\bar{\phi}}_{P}\right)+\mathrm{e}^{\bar{\lambda}_{1} \bar{x}} \overline{\bar{A}}\left(\overline{\bar{\phi}}_{P}\right)-\frac{1}{\bar{\gamma}}\right) \\
& +\frac{\Theta}{\theta_{\Delta T}}\left(1-\overline{\bar{\phi}}_{P}\right)\left(\alpha_{a 33} v_{a 31}+\alpha_{a 11}\right) \\
& \frac{\varepsilon_{c 11}\left(\bar{x}, \overline{\bar{\phi}}_{P}, \overline{\bar{\phi}}_{\text {tot }}\right)}{\overline{\bar{\phi}}_{\text {tot }}}=\frac{E_{a 11} t_{a}\left(v_{c 13} v_{c 31}-1\right)}{2 E_{c 11} t_{c}}\left(\mathrm{e}^{-\bar{\lambda}_{3} \bar{x}} \overline{\bar{D}}\left(\overline{\bar{\phi}}_{P}\right)+\mathrm{e}^{\bar{\lambda}_{3} \bar{x}} \overline{\bar{C}}\left(\overline{\bar{\phi}}_{P}\right)+\mathrm{e}^{-\bar{\lambda}_{1} \bar{x}} \overline{\bar{B}}\left(\overline{\bar{\phi}}_{P}\right)+\mathrm{e}^{\bar{\lambda}_{1} \bar{x}} \overline{\bar{A}}\left(\overline{\bar{\phi}}_{P}\right)\right)
\end{aligned}
$$

\footnotetext{
$\overline{\|\|}$ See Section 3.
} 


$$
\begin{aligned}
& +\frac{\Theta}{\theta_{\Delta T}}\left(1-\overline{\bar{\phi}}_{P}\right)\left(\alpha_{c 11}+\alpha_{c 33} v_{c 31}\right) \\
& +\frac{1}{E_{c 11} t_{c}}\left(1-v_{c 13} v_{c 31}\right)\left(\frac{E_{a 11} t_{a}}{2 \bar{\gamma}}+\frac{\overline{\bar{\phi}}_{P} \frac{\Theta}{\theta_{P}}}{1}\right)
\end{aligned}
$$

It is assumed that the total elongation is the same for the adherends. Therefore, the elongation equations are:

$$
\begin{aligned}
q_{e} & =\left(\frac{\mathrm{d} x}{\mathrm{~d} \bar{x}}\right) \int_{0}^{1} \varepsilon_{a 11}\left(\bar{x}, \overline{\bar{\phi}}_{P}, \overline{\bar{\phi}}_{\mathrm{tot}}\right) \mathrm{d} \bar{x} \\
q_{e} & =\left(\frac{\mathrm{d} x}{\mathrm{~d} \bar{x}}\right) \int_{0}^{1} \varepsilon_{c 11}\left(\bar{x}, y, \overline{\bar{\phi}}_{P}, \overline{\bar{\phi}}_{\mathrm{tot}}\right) \mathrm{d} \bar{x}
\end{aligned}
$$

As in the prior section, the sub-element elongation $q_{e}$ is defined as:

$$
q_{e}=q_{3}-q_{2}
$$

In (34), the elongation is written as a function of the dimensionless total load $\left(\overline{\bar{\phi}}_{\text {tot }}\right)$. The total load is not known a priori and must be eliminated in favor of an available quantity (the total elongation $q_{e}$ ) so that a stiffness matrix can be calculated. This is accomplished by application of the boundary conditions to the result of (34):

$$
\begin{aligned}
& \left.\left(\frac{\mathrm{d} x}{\mathrm{~d} \bar{x}}\right) \int_{0}^{\bar{x}} \varepsilon_{a 11}\left(\bar{x}, \overline{\bar{\phi}}_{P}, \overline{\bar{\phi}}_{\mathrm{tot}}\right) \mathrm{d} \bar{x}\right|_{\bar{x}=0}=0 \\
& \left.\left(\frac{\mathrm{d} x}{\mathrm{~d} \bar{x}}\right) \int_{0}^{\bar{x}} \varepsilon_{a 11}\left(\bar{x}, \overline{\bar{\phi}}_{P}, \overline{\bar{\phi}}_{\mathrm{tot}}\right) \mathrm{d} \bar{x}\right|_{\bar{x}=1}=q_{e} \\
& \left.\left(\frac{\mathrm{d} x}{\mathrm{~d} \bar{x}}\right) \int_{0}^{\bar{x}} \varepsilon_{c 11}\left(\bar{x}, \overline{\bar{\phi}}_{P}, \overline{\bar{\phi}}_{\text {tot }}\right) \mathrm{d} \bar{x}\right|_{\bar{x}=0}=0 \\
& \left.\left(\frac{\mathrm{d} x}{\mathrm{~d} \bar{x}}\right) \int_{0}^{\bar{x}} \varepsilon_{c 11}\left(\bar{x}, \overline{\bar{\phi}}_{P}, \overline{\bar{\phi}}_{\text {tot }}\right) \mathrm{d} \bar{x}\right|_{\bar{x}=1}=q_{e}
\end{aligned}
$$

Specifically, the elongation is zero when $\bar{x}=0(\bar{x}=0$ is the reference from which elongation is measured) and the total elongation is $q_{e}$ when $\bar{x}=1$. Applying these boundary conditions and solving for the total load $\left(\overline{\bar{\phi}}_{\text {tot }}\right)$ as a function of elongation $\left(q_{e}\right)$ in each strain equation, the total load can be replaced in (33):

$$
\begin{aligned}
& \overline{\bar{\phi}}_{\mathrm{tot}_{a}}=\overline{\bar{\Phi}}_{\mathrm{a}} q_{e} \\
& \overline{\bar{\phi}}_{\mathrm{tot}_{c}}=\overline{\bar{\Phi}}_{\mathrm{c}} q_{e}
\end{aligned}
$$


The intermediate terms $\left(\overline{\bar{\Phi}}_{\mathrm{a}}, \overline{\bar{\Phi}}_{\mathrm{c}}\right)$ are detailed in Appendix D. Substituting (37) into (33), the displacement field is known in terms of total elongation and the shape functions. Shape functions and their derivatives can now be written for each adherend:

$$
\begin{aligned}
u_{a}\left(\bar{x}, \overline{\bar{\phi}}_{P}, q_{e}\right) & =N_{a}\left(\bar{x}, \overline{\bar{\phi}}_{P}\right) q_{e} \\
u_{c}\left(\bar{x}, \overline{\bar{\phi}}_{P}, q_{e}\right) & =N_{c}\left(\bar{x}, \overline{\bar{\phi}}_{P}\right) q_{e} \\
B_{a}\left(\bar{x}, \overline{\bar{\phi}}_{P}\right) & =\frac{\mathrm{d}}{\mathrm{d} \bar{x}} N_{a}\left(\bar{x}, \overline{\bar{\phi}}_{P}\right) \\
B_{c}\left(\bar{x}, \overline{\bar{\phi}}_{P}\right) & =\frac{\mathrm{d}}{\mathrm{d} \bar{x}} N_{c}\left(\bar{x}, \overline{\bar{\phi}}_{P}\right)
\end{aligned}
$$

The shape functions in (38) are detailed in the Appendix E. With the established shape functions, the stiffness matrix and load vector can be integrated numerically using the following equations:

$$
\begin{gathered}
K_{e}=\frac{\sum_{\kappa} E_{\kappa 11} \int_{y_{\kappa} 0}^{y_{\kappa 1}} \int_{0}^{1} B_{\kappa}^{2}\left(\bar{x}, \overline{\bar{\phi}}_{P}\right) \mathrm{d} \bar{x} \mathrm{~d} y_{\kappa}}{l_{e}}\left[\begin{array}{cc}
1 & -1 \\
-1 & 1
\end{array}\right] \\
\mathbf{F}_{e}=P\left\{\begin{array}{c}
-1 \\
1
\end{array}\right\}+\sum_{\kappa} \alpha_{\kappa 11} E_{\kappa 11}\left(\int_{y_{0}}^{y_{1}} \int_{0}^{1} B_{\kappa}\left(\bar{x}, \overline{\bar{\phi}}_{P}\right) \mathrm{d} \bar{x} \mathrm{~d} y_{\kappa}\right) \Delta T\left\{\begin{array}{c}
-1 \\
1
\end{array}\right\}
\end{gathered}
$$

In (39) and (40), the summation includes both adherends $(\kappa=a, c)$. The sub-element stiffness matrix is adaptive to the character of the load through $\overline{\bar{\phi}}_{P}$. The strain in the adherends is related, via the material constitutive response given in (A1), to the stress fields known from (9) and Table I. These strains are related to the stiffness matrix by shape functions derivatives.

The final requirement for element calculations is knowledge of the mechanical load $(P)$ used to determine the load character $\left(\overline{\bar{\phi}}_{P}\right)$ of the lap section sub-element.

4.1.2. Calculation of the load carried across the lap section. Using the equilibrium equation for the central adherend outside the lap section, it is known that the internal load can be determined from:

$$
q_{e}=\frac{P_{2} l_{e}}{E_{a 11} t_{a}}+\alpha_{a 11} l_{e} \Delta T
$$

In terms of the displacement DOF, the above equation can be written as an additional equation in the sub-element stiffness matrix and load vector:

$$
\left[\begin{array}{lll}
\frac{E_{a 11} t_{a}}{l_{e}} & -\frac{E_{a 11} t_{a}}{l_{e}} & 1
\end{array}\right]\left\{\begin{array}{l}
q_{3} \\
q_{4} \\
P_{2}
\end{array}\right\}=\left\{\begin{array}{lll}
-E_{a 11} & t_{a} \alpha_{a 11} & \Delta T\}
\end{array}\right\}
$$

In (42), the mechanical load $\left(P_{2}\right)$ can be written as an additional degree of freedom and that is available during every increment.***

\footnotetext{
*** The current formulation of the element carries two internal load DOF $\left(P_{1}\right.$ and $\left.P_{2}\right)$ as illustrated in Figure 5 . Strictly speaking, this only requires one additional DOF.
} 


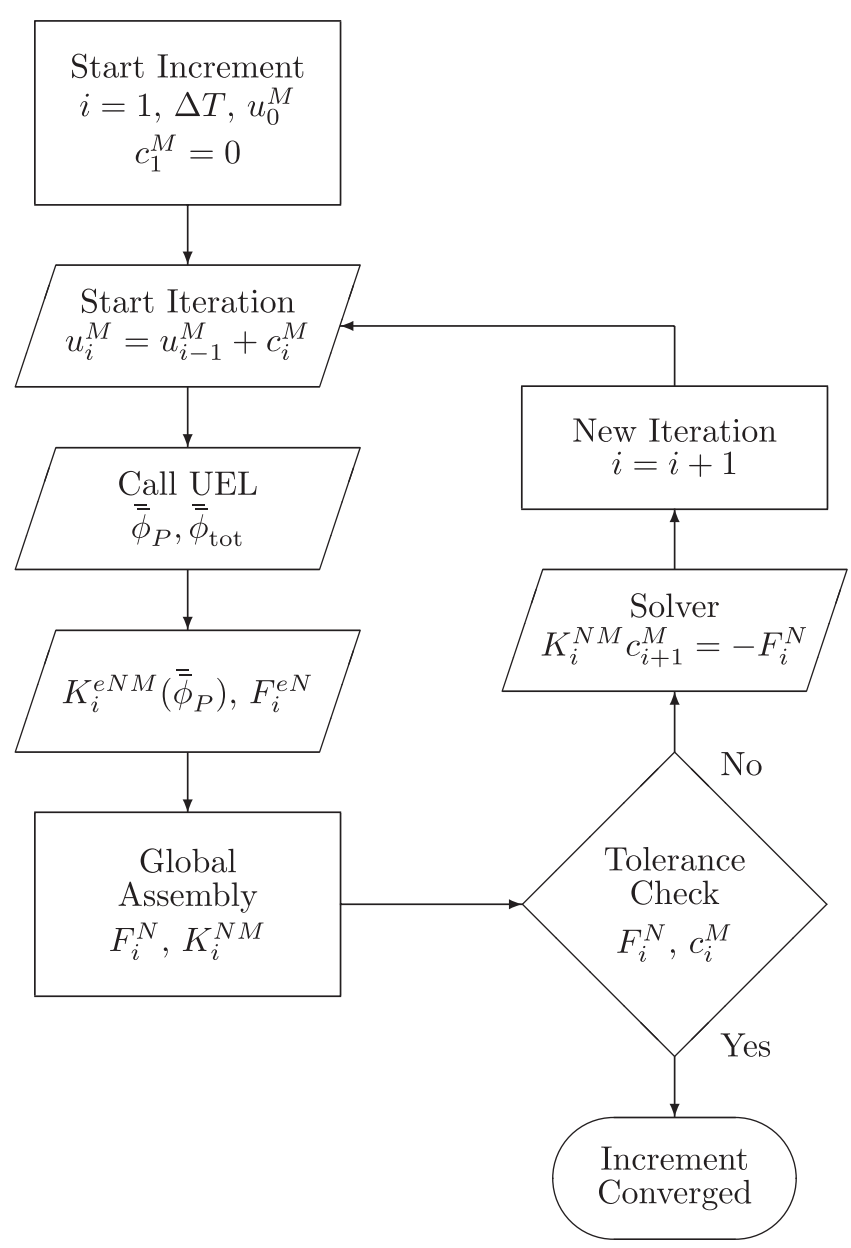

Figure 8. The solution procedure for the BJFE user element subroutine.

\subsection{The FE implementation}

The BJFE formulation requires an iterative solution since the mechanical load in the joint is not known in general. Therefore, the adaptive shape functions have been implemented as a user element subroutine (UEL) for Abaqus ${ }^{\circledR}$, a commercial non-linear FE package.

The displacement, stress and strain fields are dependent on the ratio of the mechanical to thermal load through the mechanical load fraction $\left(\overline{\bar{\phi}}_{P}\right)$. This ratio must be calculated by the BJFE. A general approach for any solution algorithm was developed in Section 4.1. In the UEL implementation, the Newton-Raphson solution algorithm allows complete elimination of the mechanical load DOF. The equations describing the algorithm are described in [39]; Figure 8 is a flowchart of the Newton-Raphson algorithm as it relates to the BJFE.

In addition to the constitutive and geometric quantities, the inputs to the BJFE are the current iteration's displacements $\left(u_{i}^{M}\right)$ and the relative temperature change $(\Delta T)$. The first step in the UEL 
is to compute the (constant) central and outer sub-element stiffnesses using (29). The central and outer sub-element load vectors are then computed using (30) and $u_{i}^{M}$. By first computing the load vectors in the outer sub-elements, the mechanical load can be used to calculate consistent shape functions and stiffnesses in the lap region. ${ }^{\dagger \dagger}$ The thermal load $(\Delta T)$ is assumed to be constant through the element and is applied as a user distributed load [27] into the BJFE. The initial iteration's value of $\Delta T$ is determined automatically by the solver based on the method chosen by the user. The default is a linear ramping of the temperature change over the step.

With knowledge of the current iteration's value of the mechanical load fraction $\left(\overline{\bar{\phi}}_{P}\right)$, the current iteration's shape functions and matrices are calculated for the lap zone sub-element. The lap zone stiffness matrix and load vector are integrated numerically using a modified midpoint rule. The modification offsets the integration point by $\frac{1}{2}$ interval so that the extremes of the joint section are included in the integration. Equal weighting is given to each interval, except that the end points have a weighting of $\frac{1}{2}$ the other intervals.

The number of integration points is defined by the user. The number is usually dictated by the desire to resolve stress gradients within the lap zone. $₫ \ddagger \ddagger$ The field quantities are calculated from Table I (at each integration point) based on the calculated $\Delta T$ and $P$ for the increment. Using this procedure, all stress and strain quantities of interest are calculated in a manner consistent with the shape function displacement field. Further, the shape functions and the resulting stiffness matrix are consistent in the Newton-Raphson algorithm and; therefore, exhibit quadratic termination [40]. Though the displacement interpolation is non-linear in $\overline{\bar{\phi}}_{P}$, the element tangent stiffness is a smooth function of $\overline{\bar{\phi}}_{P}$ over the majority of the range of $\overline{\bar{\phi}}_{P}$. Worst-case analyses converged with relative ease in all attempts.

The final task of the UEL is to assemble the sub-element stiffness matrices and load vectors into element-level matrices with 4 DOF (using standard assembly techniques [40]) and return them to the solver.

\section{BENCHMARKING}

The stress prediction of the BJFE has been compared with a conventional plane strain FE model. In the BJFE, the entire model consists of a single element. In the conventional model, a 2D mesh has been generated. Both models are based on the ASTM international (ASTM) double lap joint, [41]. Figure 2 shows the conventional mesh; Table II(a) describes the assumed geometries. The solver is Abaqus ${ }^{\circledR}$ Standard and the conventional mesh consists entirely of bilinear incompatible mode plain strain elements (CPE4I). Half of the joint is modeled due to symmetry. Loading is specified as listed in Table II(b). The mechanical load is applied far away from the lap joint and

\footnotetext{
${ }^{\dagger \dagger}$ The outer sub-element load vectors provide the current iteration's values of the mechanical loads in the lap region sub-element (provided by $P_{1}$ and $P_{2}$ in the general procedure). These forces should be equal if no body forces are applied; however, it is possible that they differ during iteration and have negligible differences after the solution completes (according to the specified convergence tolerances). Therefore, the two values are averaged for the purposes of calculating $P$ and $\overline{\bar{\phi}}_{P}$.

$\ddagger \ddagger$ The stiffness and load matrices converge with a smaller number of integration points than is usually desired for stress evaluation.
} 
Table II. Geometric and loading assumptions for model comparison: (a) ASTM double lap joint geometric features ( $\mathrm{mm}$ ) and (b) assumed loading.

\begin{tabular}{lcc}
\hline Component & Thickness & Length \\
\hline (a) & 1.6 & \\
Outer adherend & 0.2 or 1.0 & 76.2 \\
Adhesive & 3.2 & 12.7 \\
Central adherend & & 76.2 \\
(b) & & \\
Load type & & Value \\
\hline$P\left(\mathrm{Nmm}^{-1}\right)$ & & 10 \\
$\Delta T\left({ }^{\circ} \mathrm{C}\right)$ & 10 \\
\hline
\end{tabular}

Table III. Assumed material properties in FE and BJFE solutions (moduli in $\mathrm{GPa}$, expansion coefficients in $\mu \varepsilon^{\circ} \mathrm{C}$ ).

\begin{tabular}{lcccc}
\hline Material & Aluminum & Titanium & $\begin{array}{c}\text { AS4 } / 3501-6 \\
\left(0^{\circ}\right)\end{array}$ & FM300 \\
\hline$E_{11}$ & 70 & 110 & 148 & 1.98 \\
$E_{22}$ & 70 & 110 & 10.6 & 1.98 \\
$E_{33}$ & 70 & 110 & 10.6 & 1.98 \\
$G_{12}$ & 26.3 & 41.4 & 5.61 & 0.71 \\
$G_{13}$ & 26.3 & 41.4 & 5.61 & 0.71 \\
$G_{23}$ & 26.3 & 41.4 & 3.17 & 0.71 \\
$v_{12}$ & 0.33 & 0.33 & 0.30 & 0.40 \\
$v_{13}$ & 0.33 & 0.33 & 0.30 & 0.40 \\
$v_{23}$ & 0.33 & 0.33 & 0.59 & 20 \\
$\alpha_{11}$ & 23 & 9 & -0.8 & 20 \\
$\alpha_{22}$ & 23 & 9 & 29 & 20 \\
$\alpha_{33}$ & 23 & 9 & & \\
\hline
\end{tabular}

the thermal load is applied to all nodes. Displacement symmetry constraints are enforced along the mid-plane of the central adherend. Non-linear geometric stiffness is assumed.

Aluminum (AL) is the central adherend in all models; the outer adherends are titanium (TI) and AS4/3501-6 (AS4) [42]. For simplicity, the adhesive properties are assumed to be isotropic and are estimated based on Cytec FM300 adhesive. The assumed material properties are summarized in Table III.

The shear stresses from the conventional FE model are reported at the centerline of the adhesive. The centerline is the most representative location for comparison with the uniform shear stress predicted by the BJFE. The peel stress in the conventional FE model is reported at the interface between the adhesive and the central adherend. The choice of through-thickness location has a large effect on the predicted peel stress near the edge (as illustrated in Figure 3). The adhesive to central adherend interface $(a-b)$ comparison location is chosen because the BJFE model can be used as a measure of the severity of the stress field caused by the singularity at this location. The peel stress reported from the BJFE is the average peel stress through the thickness (the stress equation is evaluated at $y=t_{b} / 2$ ). 

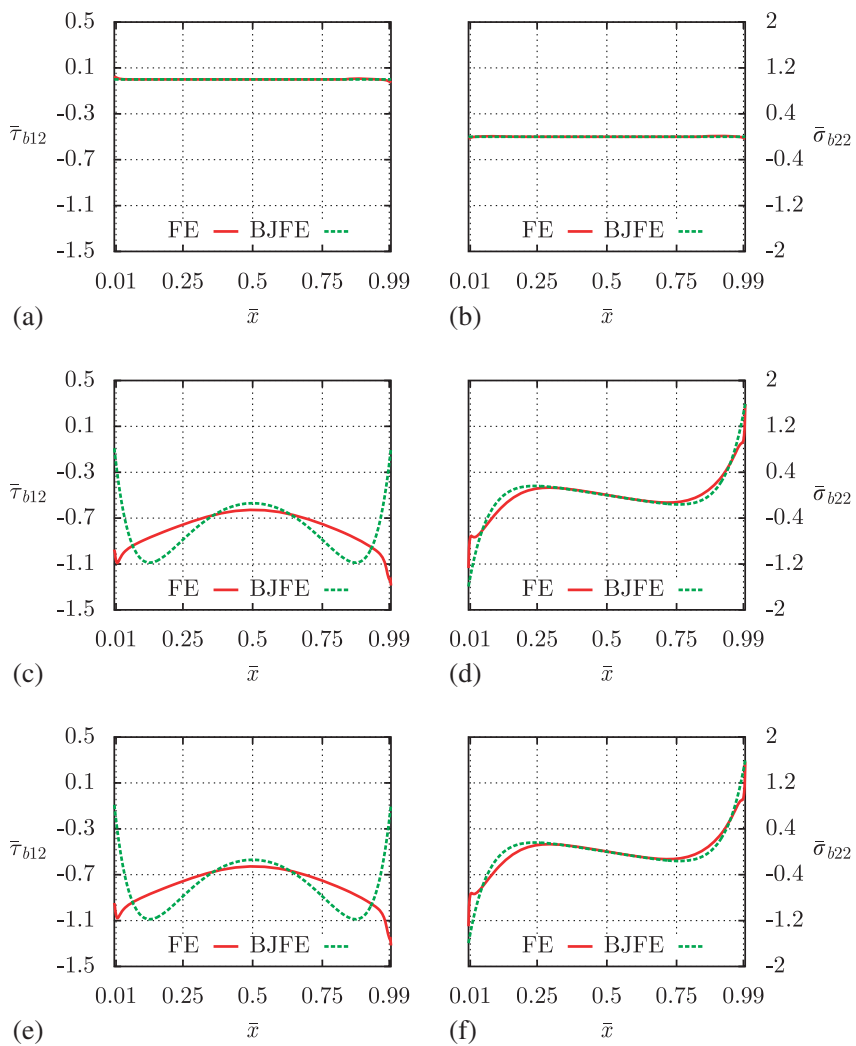

Figure 9. Continuum and BJFE models of AL-AL joint with $0.2 \mathrm{~mm}$ adhesive (MPa): (a) $\bar{\tau}_{b 12}$ due to $\bar{\phi}_{\Delta T}$; (b) $\bar{\sigma}_{b 22}$ due to $\bar{\phi}_{\Delta T}$; (c) $\bar{\tau}_{b 12}$ due to $\bar{\phi}_{P}$; (d) $\bar{\sigma}_{b 22}$ due to $\bar{\phi}_{P}$; (e) $\bar{\tau}_{b 12}$ due to $\bar{\phi}_{P}+\bar{\phi}_{\Delta T} ;$ and (f) $\bar{\sigma}_{b 22}$ due to $\bar{\phi}_{P}+\bar{\phi}_{\Delta T}$.

\subsection{Comparison of the BJFE and conventional FE models}

Figures 9-12 show stresses predicted by the conventional FE and BJFE models. Figure 9 illustrates the stress predictions for an AL-AL double lap joint. When this joint is subjected to thermal loading, as is shown in Figure 9(a) and (b), both models predict that the stress is negligible. ${ }^{\S \S}$ This stress result is intuitive, since the two adherends have identical thermal expansion coefficients (Table IV).

Figure 9(c) and (d) shows shear and peel stress predictions of the AL-AL joint subjected to mechanical loading; good agreement is exhibited in both figures. The peak shear stress predicted by the BJFE is similar to that predicted by the conventional FE model, though there is some difference in magnitude and distribution. The difference in absolute magnitude is largest near the edges where the stress singularity effects the conventional FE results. The BJFE adequately captures the magnitude of the shear result where the singular stress field does not effect the FE stress prediction. There is also a difference in the overall shear distribution. This difference is

\footnotetext{
$\S \S$ This is the special case of identical adherends. Thermal expansion of the adhesive is the primary source of loading. As reported in [37], a different analytical procedure may be more appropriate.
} 

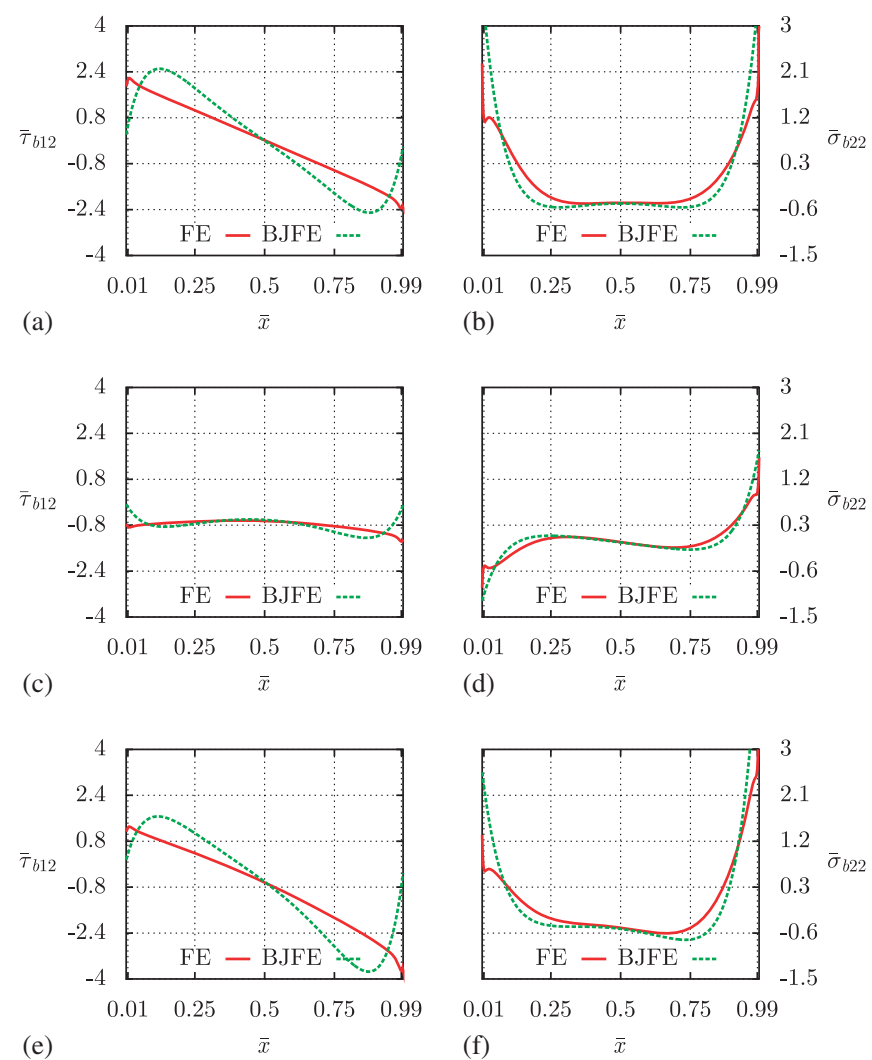

Figure 10. Continuum and BJFE models of AL-TI joint with $0.2 \mathrm{~mm}$ adhesive (MPa): (a) $\bar{\tau}_{b 12}$ due to $\bar{\phi}_{\Delta T}$; (b) $\bar{\sigma}_{b 22}$ due to $\bar{\phi}_{\Delta T} ;$ (c) $\bar{\tau}_{b 12}$ due to $\bar{\phi}_{P} ;$ (d) $\bar{\sigma}_{b 22}$ due to $\bar{\phi}_{P}$; (e) $\bar{\tau}_{b 12}$ due to $\bar{\phi}_{P}+\bar{\phi}_{\Delta T} ;$ and (f) $\bar{\sigma}_{b 22}$ due to $\bar{\phi}_{P}+\bar{\phi}_{\Delta T}$.

primarily the result of the simplifications in the virtual work solution; however, the distribution is in sufficient agreement that the difference is an unlikely primary concern when the BJFE provides an appropriate engineering approach. Similarly, the peel stress predicted by the BJFE is in adequate agreement with the conventional FE model. Neither stress quantity suffers from any mesh dependency in the BJFE prediction. Figure 9(e) and (f) shows mixed loading for the AL-AL joint, which are almost identical to the mechanical load predictions for this joint.

Figure 10 shows the stresses predicted by the conventional FE and BJFE models for an AL-TI joint. Thermal loading is non-trivial and the stress predictions resulting from it are plotted in Figure 10(a) and (b) for shear and peel. In this joint type, the predicted shear stress is in good agreement for thermal, mechanical, and mixed loading, as is shown in Figure 10(a), (c) and (e). In all cases, the peak shear stress predicted by the BJFE adequately matches the conventional FE model. The peak location is consistently found to be further from the edge in the BJFE than in the conventional FE model. The peel stress predictions in Figure 10(b), (d), and (f), also show good agreement. The stress predicted by the BJFE is similar to the conventional FE model and is representative of the (unconverged) singular peel stress result. 

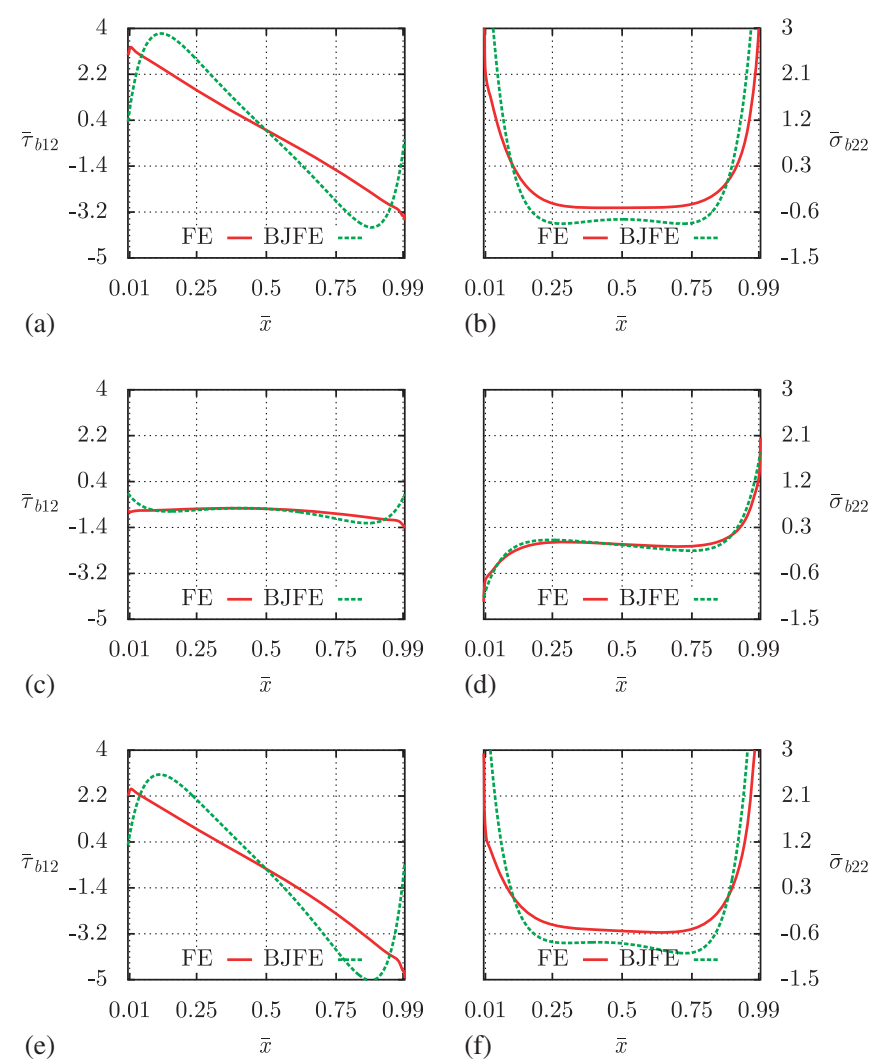

Figure 11. Continuum and BJFE models of AL-AS4 $\left(0^{\circ}\right)$ joint with $0.2 \mathrm{~mm}$ adhesive (MPa): (a) $\bar{\tau}_{b 12}$ due to $\bar{\phi}_{\Delta T}$; (b) $\bar{\sigma}_{b 22}$ due to $\bar{\phi}_{\Delta T} ;$ (c) $\bar{\tau}_{b 12}$ due to $\bar{\phi}_{P}$; (d) $\bar{\sigma}_{b 22}$ due to $\bar{\phi}_{P}$; (e) $\bar{\tau}_{b 12}$ due to $\bar{\phi}_{P}+\bar{\phi}_{\Delta T}$; and (f) $\bar{\sigma}_{b 22}$ due to $\bar{\phi}_{P}+\bar{\phi}_{\Delta T}$.

The BJFE solution is orthotropic; Figures 11 and 12 provide an example of a composite application. The figures show two AL-AS4 joints subjected to thermal, mechanical, and mixed loading. The laminate modeled in Figure 11 has fibers oriented longitudinally $\left(0^{\circ}\right)$ and the laminate modeled in Figure 12 has fibers oriented transversely $\left(90^{\circ}\right)$. Despite the unlikelihood of the $90^{\circ}$ fiber orientation (relative to the joint loading axis) in practical applications, the two figures show that the BJFE solution is in adequate agreement with the conventional FE solution in both cases and for all three load types.

By comparing Figures 9-12 to the corresponding plots in [37], it is apparent that the virtual work solution used in the BJFE is more accurate than are the simpler solutions. This is a direct result of the inclusion of additional stress terms in the virtual work solution. Based on the cumulative agreement in Figures 9-12, it can be concluded that the BJFE element adequately predicts the shear stress in a double lap joint. The peel stress predicted by the BJFE model is found to be consistently in agreement with the value of the (unconverged) singular stress field in all figures. Therefore, it can be used as a mesh independent indicator of peel stress, useful for joint-to-joint comparison. 

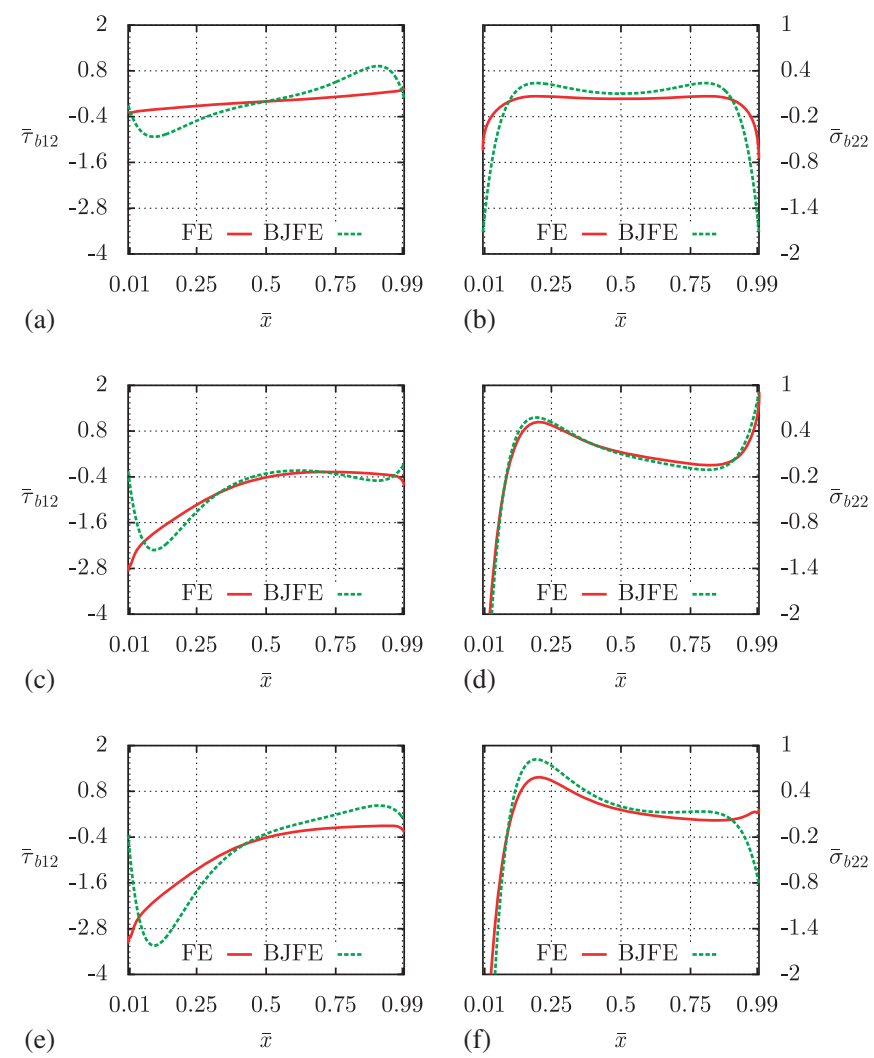

Figure 12. Continuum and BJFE models of AL-AS4 $\left(90^{\circ}\right)$ joint with $0.2 \mathrm{~mm}$ adhesive (MPa): (a) $\bar{\tau}_{b 12}$ due to $\bar{\phi}_{\Delta T}$; (b) $\bar{\sigma}_{b 22}$ due to $\bar{\phi}_{\Delta T}$; (c) $\bar{\tau}_{b 12}$ due to $\bar{\phi}_{P}$; (d) $\bar{\sigma}_{b 22}$ due to $\bar{\phi}_{P}$; (e) $\bar{\tau}_{b 12}$ due to $\bar{\phi}_{P}+\bar{\phi}_{\Delta T}$; and (f) $\bar{\sigma}_{b 22}$ due to $\bar{\phi}_{P}+\bar{\phi}_{\Delta T}$.

Table IV. Approximate size of the double lap joint FE models.

\begin{tabular}{lrrr}
\hline Model & Nodes & Elements & DOF \\
\hline CPE & 22100 & 21600 & 44300 \\
BJFE & 4 & 1 & 4 \\
\hline
\end{tabular}

\section{CONCLUSION}

In this article, an BJFE has been developed. It is capable of predicting the lap joint field quantities in the lap zone using 4 DOF. It does so without burdening the user with mesh dependency or significant meshing overhead. The BJFE is formulated by embedding an analytical solution directly within the element. Its stiffness and load response are based on adaptive non-linear shape functions that are dependent on the load character. All critical terms are formulated as functions of the dimensionless mechanical load fraction $\left(\overline{\bar{\phi}}_{P}\right)$ allowing for solution via an iterative, non-linear FE 
solver. To demonstrate its capability, the element has been implemented as a UEL in the commercial FE package Abaqus ${ }^{\circledR}$.

Based on comparison with conventional FE solutions, the 4 node BJFE is capable of adequately predicting stress in a joint due to thermal and mechanical loads. With this element, initial sizing and trade studies can be accomplished with a significantly reduced meshing investment and a reduction in computation time (compared with the conventional FE method). The element lays a foundation for advancements in bonded joint elements. Using the techniques demonstrated in this article, it is anticipated that available analytical solutions can be reformulated as application-specific bonded joint elements.

\section{APPENDIX A: PLAIN STRAIN MATERIAL CONSTITUTIVE RESPONSE}

The constitutive equations for material $\kappa$ are:

$$
\begin{aligned}
\left\{\begin{array}{l}
\varepsilon_{\kappa 11}(x) \\
\varepsilon_{\kappa 22}(x) \\
\gamma_{\kappa 12}(x)
\end{array}\right\}= & {\left[\begin{array}{ccc}
\frac{1-v_{\kappa 13} v_{\kappa 31}}{E_{\kappa 11}} & -\frac{v_{\kappa 23} v_{\kappa 31}+v_{\kappa 21}}{E_{\kappa 22}} & 0 \\
-\frac{v_{\kappa 13} v_{\kappa 32}+v_{\kappa 12}}{E_{\kappa 11}} & \frac{1-v_{\kappa 23} v_{\kappa 32}}{E_{\kappa 22}} & 0 \\
0 & 0 & \frac{1}{G_{\kappa 12}}
\end{array}\right] } \\
& \times\left\{\begin{array}{c}
\sigma_{\kappa 11}(x) \\
\sigma_{\kappa 22}(x) \\
\tau_{\kappa 12}(x)
\end{array}\right\}+\left[\begin{array}{c}
\alpha_{\kappa 33} v_{\kappa 31}+\alpha_{\kappa 11} \\
\alpha_{\kappa 33} v_{\kappa 32}+\alpha_{\kappa 22} \\
0
\end{array}\right] \Delta T
\end{aligned}
$$

A plane stress assumption could be substituted by setting the out-of-plane Poisson terms to zero $\left(v_{\kappa 13}=v_{\kappa 31}=0\right)$.

\section{APPENDIX B: EXTENDED DESCRIPTION OF THE VIRTUAL WORK CALCULATIONS}

The principles of virtual work solutions are briefly summarized below. Equilibrium relations derived in Section 3 are given in Table I as well as their associated virtual stress quantities.

In Table I, all virtual stress quantities can be written in terms of the central adherend virtual stress $\hat{\sigma}_{a 11}$. The principle of virtual work is applied using

$$
\delta W=\sum_{i} \int\left(\hat{\sigma}_{i} \varepsilon_{i}\right) \mathrm{d} V_{i}=0
$$

where $i$ represents the quantities listed in Table I for each solution. Equation (B1) applies for an arbitrary virtual stress $\hat{\sigma}_{a 11}(x)$. Plane strain constitutive relations (described in (A1)) govern each material (represented by the index $\kappa$ ). The field equations and boundary terms of the BJFE solution becomes apparent when integration of (B1) is performed by parts. 


\section{APPENDIX C: BOUNDARY CONDITIONS FOR THE BJFE SOLUTION}

The non-dimensionalized and normalized longitudinal normal stress boundary conditions for the left and right edges of the joint are:

$$
\begin{aligned}
& \overline{\bar{D}}\left(\overline{\bar{\phi}}_{P}\right)+\overline{\bar{C}}\left(\overline{\bar{\phi}}_{P}\right)+\overline{\bar{B}}\left(\overline{\bar{\phi}}_{P}\right)+\overline{\bar{A}}\left(\overline{\bar{\phi}}_{P}\right)-\frac{1}{\bar{\gamma}}=0 \\
& e^{-\bar{\lambda}_{3}} \overline{\bar{D}}\left(\overline{\bar{\phi}}_{P}\right)+e^{\bar{\lambda}_{3}} \overline{\bar{C}}\left(\overline{\bar{\phi}}_{P}\right)+e^{-\bar{\lambda}_{1}} \overline{\bar{B}}\left(\overline{\bar{\phi}}_{P}\right)+e^{\bar{\lambda}_{1}} \overline{\bar{A}}\left(\overline{\bar{\phi}}_{P}\right)-\frac{1}{\bar{\gamma}}-\frac{2 P}{E_{a 11} t_{a} \bar{\phi}_{\text {tot }}}=0
\end{aligned}
$$

The non-dimensionalized and normalized shear stress boundary conditions at the edges of the joint are:

$$
\begin{aligned}
& -\bar{\lambda}_{3} \overline{\bar{D}}\left(\overline{\bar{\phi}}_{P}\right)+\bar{\lambda}_{3} \overline{\bar{C}}\left(\overline{\bar{\phi}}_{P}\right)-\bar{\lambda}_{1} \overline{\bar{B}}\left(\overline{\bar{\phi}}_{P}\right)+\bar{\lambda}_{1} \overline{\bar{A}}\left(\overline{\bar{\phi}}_{P}\right)=0 \\
& -\bar{\lambda}_{3} e^{-\bar{\lambda}_{3}} \overline{\bar{D}}\left(\overline{\bar{\phi}}_{P}\right)+\bar{\lambda}_{3} e^{\bar{\lambda}_{3}} \overline{\bar{C}}\left(\overline{\bar{\phi}}_{P}\right)-\bar{\lambda}_{1} e^{-\bar{\lambda}_{1}} \overline{\bar{B}}\left(\overline{\bar{\phi}}_{P}\right)+\bar{\lambda}_{1} e^{\bar{\lambda}_{1}} \overline{\bar{A}}\left(\overline{\bar{\phi}}_{P}\right)=0
\end{aligned}
$$

\section{APPENDIX D: BJFE SOLUTION PARAMETERS IN TERMS OF MATERIAL PROPERTIES AND LOADS}

The following parameters are used to facilitate compact equations:

\section{D.1. Dimensionless system parameters}

Dimensionless system parameters are shown in Table DI.

\section{D.2. Dimensional material parameters}

$$
\begin{aligned}
\theta_{\beta}= & +\frac{t_{a}^{3}}{24 l^{2}}\left[\frac{\left(v_{a 12}+v_{a 13} v_{a 32}\right)}{E_{a 11}}+\frac{\left(v_{a 21}+v_{a 23} v_{a 31}\right)}{E_{a 22}}\right]-\frac{t_{a}^{2} t_{c}}{24 l^{2}}\left[\frac{\left(v_{c 12}+v_{c 13} v_{c 32}\right)}{E_{c 11}}+\frac{\left(v_{c 21}+v_{c 23} v_{c 31}\right)}{E_{c 22}}\right] \\
& +\frac{t_{a}^{2}}{4 l^{2} E_{a 11}}\left(t_{b}\left(v_{a 12}+v_{a 13} v_{a 32}\right)+\frac{t_{c}}{2}\left(v_{a 12}+v_{a 13} v_{a 32}\right)\right) \\
& +\frac{t_{a}^{2}}{4 l^{2} E_{a 22}}\left(t_{b}\left(v_{a 21}+v_{a 23} v_{a 31}\right)+\frac{t_{c}}{2}\left(v_{a 21}+v_{a 23} v_{a 31}\right)\right)-\frac{t_{a}^{2}}{8 l^{2}}\left[\frac{t_{c}}{3 G_{c 11}}+\frac{t_{b}}{G_{b 11}}\right] \\
\theta_{\gamma}= & \frac{t_{a}^{2}}{4 E_{c 11} t_{c}}\left(1-v_{c 13} v_{c 31}\right)+\frac{t_{a}}{2 E_{a 11}}\left(1-v_{a 13} v_{a 31}\right)
\end{aligned}
$$

Table DI. Dimensionless system parameters.

\begin{tabular}{lc}
\hline Load parameters & Material parameters \\
\hline $\bar{\phi}_{P}=\frac{\theta_{P} P}{\Theta}$ & $\bar{\beta}=\frac{\theta_{\beta}}{\Theta}$ \\
$\bar{\phi}_{\Delta T}=\frac{\theta_{\Delta T} \Delta T}{\Theta}$ & $\bar{\gamma}=\frac{\theta_{\gamma}}{\Theta}$ \\
\hline
\end{tabular}




\section{D.3. Dimensional load parameters}

$$
\begin{aligned}
\theta_{\Delta T} & =\frac{t_{a}}{2 E_{a 11}}\left(\alpha_{a 11}-\alpha_{c 11}+\alpha_{a 33} v_{a 31}-\alpha_{c 33} v_{c 31}\right) \\
\theta_{P} & =-\frac{t_{a}}{2 t_{c} E_{a 11} E_{c 11}}\left(1-v_{c 13} v_{c 31}\right)
\end{aligned}
$$

\section{D.4. Dimensional system parameter}

$$
\begin{aligned}
\Theta= & +\left(1-v_{a 23} v_{a 32}\right) \frac{t_{a}^{3}}{8 l^{4} E_{a 22}}\left[\frac{t_{a}^{2}}{30}+\frac{t_{a} t_{c}}{6}+\frac{t_{c}^{2}}{4}+\frac{t_{a} t_{b}}{3}+t_{b} t_{c}+t_{b}^{2}\right] \\
& +\left(1-v_{b 23} v_{b 32}\right) \frac{t_{a}^{2} t_{b}}{4 l^{4} E_{b 22}}\left[\frac{t_{c}^{2}}{4}+\frac{t_{b}^{2}}{3}+\frac{t_{b} t_{c}}{2}\right] \\
& +\left(1-v_{c 23} v_{c 32}\right) \frac{t_{a}^{2} t_{c}^{3}}{80 l^{4} E_{c 22}}
\end{aligned}
$$

\section{D.5. Denominators of the elimination coefficients}

The denominators from (37) are:

$$
\begin{aligned}
\bar{\Xi}_{a}= & +l_{e} \bar{\lambda}_{1}\left(1-v_{a 13} v_{a 31}\right)\left(e^{\bar{\lambda}_{3}+\bar{\lambda}_{1}}-e^{\bar{\lambda}_{1}}\right) \overline{\bar{D}}\left(\overline{\bar{\phi}}_{P}\right) \\
& +l_{e} \bar{\lambda}_{1}\left(1-v_{a 13} v_{a 31}\right)\left(e^{2 \bar{\lambda}_{3}+\bar{\lambda}_{1}}-e^{\bar{\lambda}_{3}+\bar{\lambda}_{1}}\right) \overline{\bar{C}}\left(\overline{\bar{\phi}}_{P}\right) \\
& +l_{e} \bar{\lambda}_{3}\left(1-v_{a 13} v_{a 31}\right)\left(e^{\bar{\lambda}_{3}+\bar{\lambda}_{1}}-e^{\bar{\lambda}_{3}}\right) \overline{\bar{B}}\left(\overline{\bar{\phi}}_{P}\right) \\
& +l_{e} \bar{\lambda}_{3}\left(1-v_{a 13} v_{a 31}\right)\left(e^{\bar{\lambda}_{3}+2 \bar{\lambda}_{1}}-e^{\bar{\lambda}_{3}+\bar{\lambda}_{1}}\right) \overline{\bar{A}}\left(\overline{\bar{\phi}}_{P}\right) \\
& -l_{e} \bar{\lambda}_{1} \bar{\lambda}_{3} e^{\bar{\lambda}_{3}+\bar{\lambda}_{1}}\left(\frac{\left(1-v_{a 13} v_{a 31}\right)}{\bar{\gamma}}-\chi_{\Delta T}\left(1-\overline{\bar{\phi}}_{P}\right)\left(\alpha_{a 11}+\alpha_{a 33} v_{a 31}\right)\right) \\
\bar{\Xi}_{c}= & -\frac{E_{a 11} t_{a}}{2 E_{c 11} t_{c}} l_{e} \bar{\lambda}_{1}\left(1-v_{c 13} v_{c 31}\right)\left(e^{\bar{\lambda}_{1}}+e^{\bar{\lambda}_{3}+\bar{\lambda}_{1}}\right) \overline{\bar{D}}\left(\overline{\bar{\phi}}_{P}\right) \\
& -\frac{E_{a 11} t_{a}}{2 E_{c 11} t_{c}} l_{e} \bar{\lambda}_{1}\left(1-v_{c 13} v_{c 31}\right)\left(e^{2 \bar{\lambda}_{3}+\bar{\lambda}_{1}}-e^{\bar{\lambda}_{3}+\bar{\lambda}_{1}}\right) \overline{\bar{C}}_{\left(\bar{\phi}_{P}\right)} \\
& -\frac{E_{a 11} t_{a}}{2 E_{c 11} l_{c}} l_{e} \bar{\lambda}_{3}\left(1-v_{c 13} v_{c 31}\right)\left(e^{\bar{\lambda}_{3}}+e^{\bar{\lambda}_{3}+\bar{\lambda}_{1}}\right) \overline{\bar{B}}\left(\overline{\bar{\phi}}_{P}\right)
\end{aligned}
$$




$$
\begin{aligned}
& -\frac{E_{a 11} t_{a}}{2 E_{c 11} t_{c}} l_{e} \bar{\lambda}_{3}\left(1-v_{c 13} v_{c 31}\right)\left(e^{\bar{\lambda}_{3}+2 \bar{\lambda}_{1}}-e^{\bar{\lambda}_{3}+\bar{\lambda}_{1}}\right) \overline{\bar{A}}\left(\overline{\bar{\phi}}_{P}\right) \\
& +\frac{l_{e} \bar{\lambda}_{1} \bar{\lambda}_{3} e^{\bar{\lambda}_{3}+\bar{\lambda}_{1}}}{2 E_{c 11} t_{c}}\left(\frac{\left(1-v_{c 13} v_{c 31}\right)}{\bar{\gamma}}\left(E_{a 11} t_{a}+2 \bar{\gamma} \overline{\bar{\phi}}_{P} \chi_{P}\right)\right) \\
& +\frac{l_{e} \bar{\lambda}_{1} \bar{\lambda}_{3} e^{\bar{\lambda}_{3}+\bar{\lambda}_{1}}}{2 E_{c 11} t_{c}}\left(\chi_{\Delta T}\left(1-\overline{\bar{\phi}}_{P}\right)\left(\alpha_{c 11}+\alpha_{c 33} v_{c 31}\right)\right)
\end{aligned}
$$

\section{D.6. Simplification coefficients}

$$
\begin{aligned}
& \overline{\bar{\Phi}}_{\mathrm{a}}=\frac{\bar{\lambda}_{1} \bar{\lambda}_{3} e^{\bar{\lambda}_{3}+\bar{\lambda}_{1}}}{\bar{\Xi}_{\mathrm{a}}} \\
& \overline{\bar{\Phi}}_{\mathrm{c}}=\frac{\bar{\lambda}_{1} \bar{\lambda}_{3} e^{\bar{\lambda}_{3}+\bar{\lambda}_{1}}}{\overline{\bar{\Xi}}_{\mathrm{c}}} \\
& \overline{\bar{\Xi}}_{\mathrm{a}}=+l_{e} \bar{\lambda}_{1}\left(1-v_{a 13} v_{a 31}\right)\left(e^{\bar{\lambda}_{3}+\bar{\lambda}_{1}}-e^{\bar{\lambda}_{1}}\right) \overline{\bar{D}}\left(\overline{\bar{\phi}}_{P}\right) \\
& +l_{e} \bar{\lambda}_{1}\left(1-v_{a 13} v_{a 31}\right)\left(e^{2 \bar{\lambda}_{3}+\bar{\lambda}_{1}}-e^{\bar{\lambda}_{3}+\bar{\lambda}_{1}}\right) \overline{\bar{C}}\left(\overline{\bar{\phi}}_{P}\right) \\
& +l_{e} \bar{\lambda}_{3}\left(1-v_{a 13} v_{a 31}\right)\left(e^{\bar{\lambda}_{3}+\bar{\lambda}_{1}}-e^{\bar{\lambda}_{3}}\right) \overline{\bar{B}}\left(\overline{\bar{\phi}}_{P}\right) \\
& +l_{e} \bar{\lambda}_{3}\left(1-v_{a 13} v_{a 31}\right)\left(e^{\bar{\lambda}_{3}+2 \bar{\lambda}_{1}}-e^{\bar{\lambda}_{3}+\bar{\lambda}_{1}}\right) \overline{\bar{A}}\left(\overline{\bar{\phi}}_{P}\right) \\
& -l_{e} \bar{\lambda}_{1} \bar{\lambda}_{3} e^{\bar{\lambda}_{3}+\bar{\lambda}_{1}}\left(\frac{\left(1-v_{a 13} v_{a 31}\right)}{\bar{\gamma}}-\frac{\Theta}{\theta_{\Delta T}}\left(1-\overline{\bar{\phi}}_{P}\right)\left(\alpha_{a 11}+\alpha_{a 33} v_{a 31}\right)\right) \\
& \overline{\bar{\Xi}}_{\mathrm{c}}=-\frac{E_{a 11} t_{a}}{2 E_{c 11} t_{c}} l_{e} \bar{\lambda}_{1}\left(1-v_{c 13} v_{c 31}\right)\left(e^{\bar{\lambda}_{1}}+e^{\bar{\lambda}_{3}+\bar{\lambda}_{1}}\right) \overline{\bar{D}}\left(\overline{\bar{\phi}}_{P}\right) \\
& -\frac{E_{a 11} t_{a}}{2 E_{c 11} t_{c}} l_{e} \bar{\lambda}_{1}\left(1-v_{c 13} v_{c 31}\right)\left(e^{2 \bar{\lambda}_{3}+\bar{\lambda}_{1}}-e^{\bar{\lambda}_{3}+\bar{\lambda}_{1}}\right) \overline{\bar{C}}\left(\overline{\bar{\phi}}_{P}\right) \\
& -\frac{E_{a 11} t_{a}}{2 E_{c 11} t_{c}} l_{e} \bar{\lambda}_{3}\left(1-v_{c 13} v_{c 31}\right)\left(e^{\bar{\lambda}_{3}}+e^{\bar{\lambda}_{3}+\bar{\lambda}_{1}}\right) \overline{\bar{B}}\left(\overline{\bar{\phi}}_{P}\right) \\
& -\frac{E_{a 11} t_{a}}{2 E_{c 11} t_{c}} l_{e} \bar{\lambda}_{3}\left(1-v_{c 13} v_{c 31}\right)\left(e^{\bar{\lambda}_{3}+2 \bar{\lambda}_{1}}-e^{\bar{\lambda}_{3}+\bar{\lambda}_{1}}\right) \overline{\bar{A}}\left(\overline{\bar{\phi}}_{P}\right)
\end{aligned}
$$




$$
\begin{aligned}
& +\frac{l_{e} \bar{\lambda}_{1} \bar{\lambda}_{3} e^{\bar{\lambda}_{3}+\bar{\lambda}_{1}}}{2 E_{c 11} t_{c}}\left(\frac{\left(1-v_{c 13} v_{c 31}\right)}{\bar{\gamma}}\left(E_{a 11} t_{a}+2 \bar{\gamma} \overline{\bar{\phi}}_{P} \frac{\Theta}{\theta_{P}}\right)\right) \\
& +\frac{l_{e} \bar{\lambda}_{1} \bar{\lambda}_{3} e^{\bar{\lambda}_{3}+\bar{\lambda}_{1}}}{2 E_{c 11} t_{c}}\left(\frac{\Theta}{\theta_{\Delta T}}\left(1-\overline{\bar{\phi}}_{P}\right)\left(\alpha_{c 11}+\alpha_{c 33} v_{c 31}\right)\right)
\end{aligned}
$$

D.7. System parameters $\bar{\lambda}_{[13]}$ in terms of the orthotropic material properties

$$
\begin{aligned}
\bar{\lambda}^{2}= & \frac{ \pm \sqrt{\frac{9 E_{b 22}^{2} l^{4}}{4 G_{b 12}^{2} t_{b}^{4}\left(v_{b 23} v_{b 32}-1\right)^{2}}-\frac{12 E_{b 22} l^{4}\left(E_{a 11} t_{a} v_{c 13} v_{c 31}+2 E_{c 11} t_{c} v_{a 13} v_{a 31}-2 E_{c 11} t_{c}-E_{a 11} t_{a}\right)}{E_{a 11} E_{c 11} t_{a} t_{b}^{3} t_{c}\left(v_{b 23} v_{b 32}-1\right)}}}{2} \\
& -\frac{3 E_{b 22} l^{2}}{4 G_{b 12} t_{b}^{2}\left(v_{b 23} v_{b 32}-1\right)}
\end{aligned}
$$

D.8. $\mu$ parameters for the BJFE solution basis functions

$$
\begin{aligned}
& \mu_{A_{T}}=\frac{\bar{\lambda}_{3}\left(e^{\bar{\lambda}_{3}}-1\right)}{\bar{\gamma}} \\
& \mu_{B_{T}}=\frac{e^{\bar{\lambda}_{1}} \bar{\lambda}_{3}\left(e^{\bar{\lambda}_{3}}-1\right)}{\bar{\gamma}} \\
& \mu_{C_{T}}=-\frac{\bar{\lambda}_{1}\left(e^{\bar{\lambda}_{1}}-1\right)}{\bar{\gamma}} \\
& \mu_{D_{T}}=-\frac{\bar{\lambda}_{1}\left(e^{\bar{\lambda}_{1}}-1\right) e^{\bar{\lambda}_{3}}}{\bar{\gamma}} \\
& \mu_{A_{P}}=-\left(\bar{\lambda}_{3} e^{2 \bar{\lambda}_{3}+\bar{\lambda}_{1}}-\bar{\lambda}_{1} e^{2 \bar{\lambda}_{3}+\bar{\lambda}_{1}}+2 \bar{\lambda}_{1} e^{\bar{\lambda}_{3}}-e^{\bar{\lambda}_{1}} \bar{\lambda}_{3}-\bar{\lambda}_{1} e^{\bar{\lambda}_{1}}\right) \\
& \mu_{B_{P}}=e^{\bar{\lambda}_{1}}\left(-2 \bar{\lambda}_{1} e^{\bar{\lambda}_{3}+\bar{\lambda}_{1}}+\bar{\lambda}_{3} e^{2 \bar{\lambda}_{3}}+\bar{\lambda}_{1} e^{2 \bar{\lambda}_{3}}-\bar{\lambda}_{3}+\bar{\lambda}_{1}\right) \\
& \mu_{C_{P}}=\frac{\bar{\lambda}_{1}\left(\bar{\lambda}_{3} e^{\bar{\lambda}_{3}+2 \bar{\lambda}_{1}}-\bar{\lambda}_{1} e^{\bar{\lambda}_{3}+2 \bar{\lambda}_{1}}+\bar{\lambda}_{3} e^{\bar{\lambda}_{3}}+\bar{\lambda}_{1} e^{\bar{\lambda}_{3}}-2 e^{\left.\bar{\lambda}_{1} \bar{\lambda}_{3}\right)}\right.}{\bar{\lambda}_{3}} \\
& \mu_{D_{P}}=-\frac{\bar{\lambda}_{1} e^{\bar{\lambda}_{3}}\left(2 \bar{\lambda}_{3} e^{\bar{\lambda}_{3}+\bar{\lambda}_{1}}-e^{\left.2 \bar{\lambda}_{1} \bar{\lambda}_{3}-\bar{\lambda}_{3}-\bar{\lambda}_{1} e^{2 \bar{\lambda}_{1}}+\bar{\lambda}_{1}\right)}\right.}{\bar{\lambda}_{3}}
\end{aligned}
$$




$$
\begin{aligned}
& \mu_{1}=\bar{\lambda}_{3} e^{\bar{\lambda}_{3}+\bar{\lambda}_{1}}-\bar{\lambda}_{1} e^{\bar{\lambda}_{3}+\bar{\lambda}_{1}}+\bar{\lambda}_{3} e^{\bar{\lambda}_{3}}+\bar{\lambda}_{1} e^{\bar{\lambda}_{3}}-e^{\bar{\lambda}_{1}} \bar{\lambda}_{3}-\bar{\lambda}_{3}-\bar{\lambda}_{1} e^{\bar{\lambda}_{1}}+\bar{\lambda}_{1} \\
& \mu_{2}=\bar{\lambda}_{3} e^{\bar{\lambda}_{3}+\bar{\lambda}_{1}}-\bar{\lambda}_{1} e^{\bar{\lambda}_{3}+\bar{\lambda}_{1}}-\bar{\lambda}_{3} e^{\bar{\lambda}_{3}}-\bar{\lambda}_{1} e^{\bar{\lambda}_{3}}+e^{\bar{\lambda}_{1}} \bar{\lambda}_{3}-\bar{\lambda}_{3}+\bar{\lambda}_{1} e^{\bar{\lambda}_{1}}+\bar{\lambda}_{1} \\
& \mu_{3}=\frac{E_{c 11} \bar{\lambda}_{3} t_{b}^{3} t_{c}\left(v_{b 23} v_{b 32}-1\right)}{3 E_{b 22} l^{4}\left(v_{c 13} v_{c 31}-1\right)}
\end{aligned}
$$

\section{APPENDIX E: BJFE SHAPE FUNCTIONS AND DERIVATIVES WITHIN}

\section{THE LAP REGION}

$$
\begin{aligned}
& \frac{N_{a}\left(\bar{x}, \overline{\bar{\phi}}_{P}\right)}{l_{e} \overline{\bar{\Phi}}_{\mathrm{a}}}=-\left(1-v_{a 13} v_{a 31}\right) \\
& \cdot\left(\frac{e^{-\bar{\lambda}_{3} \bar{x} \overline{\bar{D}}\left(\overline{\bar{\phi}}_{P}\right)}}{\bar{\lambda}_{3}}+\frac{-e^{\bar{\lambda}_{3} \bar{x}} \overline{\bar{C}}\left(\overline{\bar{\phi}}_{P}\right)}{\bar{\lambda}_{3}}+\frac{e^{-\bar{\lambda}_{1} \bar{x}} \overline{\bar{B}}\left(\overline{\bar{\phi}}_{P}\right)}{\bar{\lambda}_{1}}+\frac{-e^{\bar{\lambda}_{1} \bar{x}} \overline{\bar{A}}_{\left(\bar{\phi}_{P}\right)}}{\bar{\lambda}_{1}}+\frac{\bar{x}}{\bar{\gamma}}\right) \\
& +\bar{x} \chi_{\Delta T}\left(1-\overline{\bar{\phi}}_{P}\right)\left(\alpha_{a 33} v_{a 31}+\alpha_{a 11}\right) \\
& \frac{N_{c}\left(\bar{x}, \overline{\bar{\phi}}_{P}\right)}{l \overline{\bar{\Phi}}_{\mathrm{c}}}=\frac{E_{a 11} t_{a}\left(1-v_{c 13} v_{c 31}\right)}{2 E_{c 11} t_{c}} \\
& \cdot\left(\frac{e^{-\bar{\lambda}_{3} \bar{x} \overline{\bar{D}}\left(\overline{\bar{\phi}}_{P}\right)}}{\bar{\lambda}_{3}}-\frac{e^{\bar{\lambda}_{3} \bar{x}} \overline{\bar{C}}\left(\overline{\bar{\phi}}_{P}\right)}{\bar{\lambda}_{3}}+\frac{e^{-\bar{\lambda}_{1} \bar{x}} \overline{\bar{B}}\left(\overline{\bar{\phi}}_{P}\right)}{\bar{\lambda}_{1}}-\frac{e^{\bar{\lambda}_{1} \bar{x}} \overline{\bar{A}}\left(\overline{\bar{\phi}}_{P}\right)}{\bar{\lambda}_{1}}\right) \\
& +\frac{\bar{x}\left(1-v_{c 13} v_{c 31}\right)}{E_{c 11} t_{c}}\left(\overline{\bar{\phi}}_{P} \chi_{P}+\frac{E_{a 11} t_{a}}{2 \bar{\gamma}}\right) \\
& +\bar{x} \chi_{\Delta T}\left(1-\overline{\bar{\phi}}_{P}\right)\left(\alpha_{c 33} v_{c 31}+\alpha_{c 11}\right) \\
& \frac{B_{a}\left(\bar{x}, \overline{\bar{\phi}}_{P}\right)}{l_{e} \overline{\bar{\Phi}}_{\mathrm{a}}}=\left(1-v_{a 13} v_{a 31}\right) \\
& \cdot\left(e^{-\bar{\lambda}_{3} \bar{x}} \overline{\bar{D}}\left(\overline{\bar{\phi}}_{P}\right)+e^{\bar{\lambda}_{3} \bar{x}} \overline{\bar{C}}\left(\overline{\bar{\phi}}_{P}\right)+e^{-\bar{\lambda}_{1} \bar{x}} \overline{\bar{B}}\left(\overline{\bar{\phi}}_{P}\right)+e^{\bar{\lambda}_{1} \bar{x}} \overline{\bar{A}}\left(\overline{\bar{\phi}}_{P}\right)-\frac{1}{\bar{\gamma}}\right) \\
& +\chi_{\Delta T}\left(1-\overline{\bar{\phi}}_{P}\right)\left(\alpha_{a 33} v_{a 31}+\alpha_{a 11}\right)
\end{aligned}
$$




$$
\begin{aligned}
& \frac{B_{c}\left(\bar{x}, \overline{\bar{\phi}}_{P}\right)}{l_{e} \overline{\bar{\Phi}}_{\mathrm{c}}}=-\left(1-v_{c 13} v_{c 31}\right) \frac{E_{a 11} t_{a}}{2 E_{c 11} t_{c}} \\
& \cdot\left(e^{-\bar{\lambda}_{3} \bar{x}} \overline{\bar{D}}\left(\overline{\bar{\phi}}_{P}\right)+e^{\bar{\lambda}_{3} \bar{x}} \overline{\bar{C}}\left(\overline{\bar{\phi}}_{P}\right)+e^{-\bar{\lambda}_{1} \bar{x}} \overline{\bar{B}}\left(\overline{\bar{\phi}}_{P}\right)+e^{\bar{\lambda}_{1} \bar{x}} \overline{\bar{A}}\left(\overline{\bar{\phi}}_{P}\right)-\frac{1}{\bar{\gamma}}\right) \\
& +\frac{\overline{\bar{\phi}}_{P} \chi_{P}\left(1-v_{c 13} v_{c 31}\right)}{E_{c 11} t_{c}}+\chi_{\Delta T}\left(1-\overline{\bar{\phi}}_{P}\right)\left(\alpha_{c 33} v_{c 31}+\alpha_{c 11}\right)
\end{aligned}
$$

\section{NOMENCLATURE}

$\alpha_{\kappa i i}$

$\bar{a}, \bar{b}, \overline{\bar{a}}, \overline{\bar{b}}$

$\bar{A}, \bar{B}, \bar{C}, \bar{D}$

$\overline{\bar{A}}, \overline{\bar{B}}, \overline{\bar{C}}, \overline{\bar{D}}$

$B_{a}, B_{c}$

$F_{i}^{N}$

$u_{i}^{M}$

$\chi_{\Delta T}, \chi_{P}$

$\Delta T$

$E_{\kappa i i}$

$F_{\text {el }}$

$F$

$G_{b i j}$

$\hat{\sigma}_{\kappa 11(x)}$

$\hat{\sigma}_{\kappa 22(x, y)}$

$\hat{\tau}_{\kappa 12(x)}$

$c_{0}, c_{1}, d_{1}, d_{0}$

$l_{e}$

$\mu_{A_{\Delta T}}, \mu_{B_{\Delta T}}, \mu_{C_{\Delta T}}, \mu_{D_{\Delta T}}$

$\mu_{A_{P}}, \mu_{B_{P}}, \mu_{C_{P}}, \mu_{D_{P}}$

$\mu_{1}, \mu_{2}, \mu_{3}, \mu_{4}, \mu_{5}$

$N_{a}, N_{c}$

$\theta_{\beta}, \theta_{\gamma}, \theta_{\Delta T}, \theta_{P}, \Theta$

$v_{\kappa i j}$

$\beta, \gamma, \omega$

$\bar{\beta}, \bar{\gamma}, \bar{\omega}, \bar{\lambda}_{1}, \bar{\lambda}_{3}$

$\bar{\phi}_{\mathrm{aR}}, \bar{\phi}_{\mathrm{cR}}$

$\overline{\bar{\phi}}_{P}, \overline{\bar{\psi}}_{P}$

$\overline{\bar{\phi}}, \overline{\bar{\psi}}$

$\bar{\phi}_{P}, \bar{\psi}_{P}$ orthotropic thermal expansion coefficient of component $\kappa$

dimensionless basis coefficients

dimensionless basis coefficients

dimensionless basis coefficients

element shape function derivatives

force vector in increment $i$

displacement vector in increment $i$

simplification coefficients

temperature change from a reference temperature

orthotropic engineering moduli of component $\kappa$

element force vector

force in an end post

orthotropic shear modulii of the adhesive

longitudinal virtual stress in component $\kappa$

transverse virtual stress in component $\kappa$

shear virtual stress in component $\kappa$

integration constants

length of current sub-element

simplification coefficients

simplification coefficients

simplification coefficients

element shape functions

simplification coefficients

Poisson's ratios of component $\kappa$

lap joint system parameters

dimensionless lap joint system parameters

dimensionless thermal to mechanical load ratios

dimensionless mechanical load fractions

dimensionless total load parameters

dimensionless mechanical load parameters 


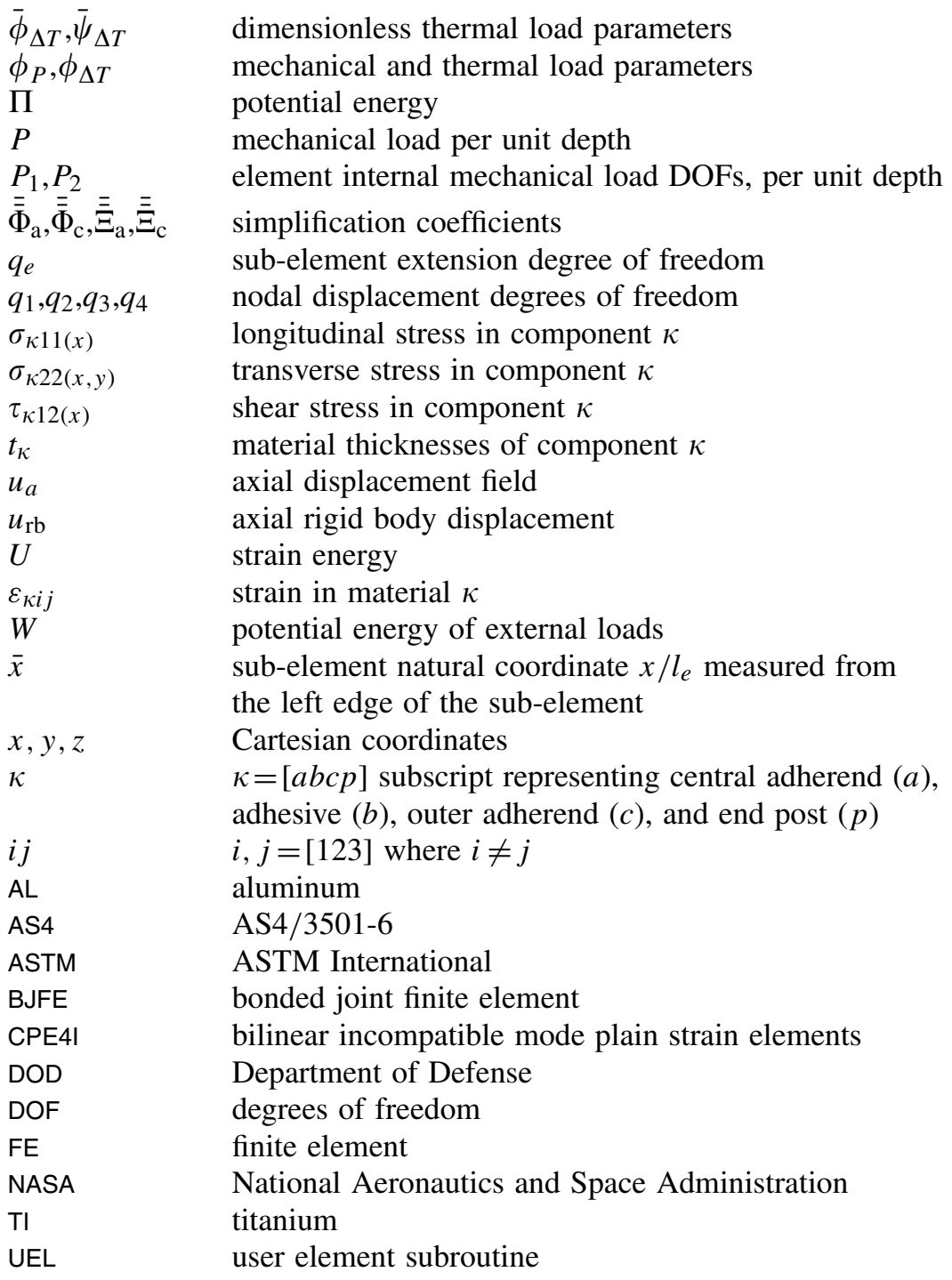

\section{ACKNOWLEDGEMENTS}

This work was supported by the Space Vehicle Technology Institute under grant NCC3-989 jointly funded by NASA and the DOD within the NASA Constellation University Institutes Project. Claudia Meyer was the project manager and Dr Stanley Smeltzer III and Dr H. Kevin Rivers were project monitors.

\section{REFERENCES}

1. Wooley GR, Carver DR. Stress concentration factors for bonded lap joints. Journal of Aircraft 1971; 8:817-820.

2. Adams RD, Peppiatt NA. Stress analysis of adhesive-bonded lap joints. Journal of Strain Analysis 1974; 9(3):185-196. 
3. Dávila CG. Mixed-mode decohesion elements for analysis of progressive delamination. 42nd AIAA/ASME/ ASCE/AHS/ASC Structures, Structural Dynamics and Materials Conference, Seattle, WA, 2001.

4. Kafkalidis MS, Thouless MD. The effects of geometry and material properties on the fracture of single lap-shear joints. International Journal of Solids and Structures 2002; 39(17):4367-4383.

5. Camanho PP, Dávila CG. Mixed-mode decohesion finite elements for the simulation of delamination in composite materials. Technical Report TM-2002-211737, NASA, 2002.

6. Xie De, Waas AM, Shahwan KW, Schroeder JA, Boeman RG. Fracture criterion for kinking cracks in a tri-material adhesively bonded joint under mixed mode loading. Engineering Fracture Mechanics 2005; 72(16):2487-2504.

7. Li S, Thouless MD, Waas AM, Schroeder JA, Zavattieri PD. Use of a cohesive-zone model to analyze the fracture of a fiber-reinforced polymer-matrix composite. Composites Science and Technology 2005; 65(3-4):537-549.

8. Li S, Thouless MD, Waas AM, Schroeder JA, Zavattieri PD. Competing failure mechanisms in mixed-mode fracture of an adhesively bonded polymer-matrix composite. International Journal of Adhesion and Adhesives 2006; 26(8):609-616.

9. Valoroso N, Champaney L. A damage-mechanics-based approach for modelling decohesion in adhesively bonded assemblies. Engineering Fracture Mechanics 2006; 73(18):2774-2801.

10. Xie De, Waas AM. Discrete cohesive zone model for mixed-mode fracture using finite element analysis. Engineering Fracture Mechanics 2006; 73(13):1783-1796.

11. Gustafson PA, Waas AM. T650/AFR-PE-4/FM680-1 mode I critical energy release rate at high temperatures: experiments and numerical models. Proceedings of the AIAA/ASME/ASCE/AHS/ASC 48th Structures, Structural Dynamics, and Materials Conference, Honolulu, HI, vol. 2007-2305, American Institute of Aeronautics and Astronautics, 23-26 April 2007.

12. Gustafson PA, Waas AM. Efficient and robust traction laws for the modeling of adhesively bonded joints. Proceedings of the AIAA/ASME/ASCE/AHS/ASC 49th Structures, Structural Dynamics, and Materials Conference, Schaumburg, IL, vol. 2008-1847, American Institute of Aeronautics and Astronautics, 7-10 April 2008.

13. Weerts U, Kossira H. Mixed mode fracture characterization of adhesive joints. ICAS Congress. International Congress Aeronautical Sciences, Harrogate (GB), 2000; 451.1-451.9.

14. Aydemir A, Gunay D. The fuzzy finite element stress analysis of adhesive-bonded single lap joints. Turkish Journal of Engineering and Environment Sciences 2003; 121-127.

15. Koutsourelakis PS, Kuntiyawichai K, Schueller GI. Effect of material uncertainties on fatigue life calculations of aircraft fuselages: a cohesive element model. Engineering Fracture Mechanics 2006; 73(9):1202-1219.

16. Gillespie Jr JW, Carlsson LA, Pipes RB. Finite element analysis of the end notched flexure specimen for measuring mode ii fracture toughness. Composites Science and Technology 1986; 27(3):177-197.

17. Wang JT, Raju IS, Sleight DW. Fracture mechanics analyses of composite skin-stiffener debond configurations with shell elements. Number 94-1389CP, 1994.

18. Glaessgen EH, Raju IS, Poe Jr CC. Fracture mechanics analysis of stitched stiffener-skin debonding. 39th AIAA/ASME/ASCE/AHS/ASC Structures, Structural Dynamics, and Materials Conference, Long Beach, CA, vol. 98-2022, 1998.

19. Krueger R. Virtual crack closure technique: history, approach, and applications. Applied Mechanics Reviews 2004; 57(2):109-143.

20. Xie De, Waas AM, Shahwan KW, Schroeder JA, Boeman RG. Computation of energy release rates for kinking cracks based on virtual crack closure technique. Computer Modeling in Engineering and Sciences 2004; 6: 515-524.

21. Xie De, Salvi AG, Waas AM, Caliskan A. Discrete cohesive zone model to simulate static fracture in carbon fiber textile composites. 46th AIAA/ASME/ASCE/AHS/ASC Structures, Structrual Dynamics and Materials Conference, Austin, TX, 2005.

22. Xie De, Biggers Jr SB. Progressive crack growth analysis using interface element based on the virtual crack closure technique. Finite Elements in Analysis and Design 2006; 42(11):977-984.

23. Goncalves JPM, de Moura MFSF, de Castro PMST. A three-dimensional finite element model for stress analysis of adhesive joints. International Journal of Adhesion and Adhesives 2002; 22(5):357-365.

24. Goyal VK, Johnson ER, Cassino C. Computational model for progressive failure of adhesivley bonded joints. 44th AIAA/ASME/ASCE/AHS/ASC Structures, Structrual Dynamics and Materials Conference, Norfolk, VA, 2003.

25. Munoz JJ, Galvanetto U, Robinson P. On the numerical simulation of fatigue driven delamination with interface elements. International Journal of Fatigue 2006; 28(10):1136-1146. 
26. Ban C-S, Lee Y-H, Choi J-H, Kweon J-H. Strength prediction of adhesive joints using the modified damage zone theory. Composite Structures 2008; 86(1-3):96-100.

27. Inc Abaqus. Abaqus User Manual v6.6. Electronic Version, 2006.

28. Alpha STAR Corp. GENOA. 5199 East Pacific Coast Highway, Long Beach, CA, U.S.A., 2008.

29. Sandia National Laboratory. Tahoe User Guide 3.4.1 Edition, May 2003.

30. Oterkus E, Barut A, Madenci E, Smeltzer III SS, Ambur DR. Non-linear analysis of bonded composite single-lap joints. Proceedings of the AIAA/ASME/ASCE/AHS/ASC 45th Structures, Structural Dynamics, and Materials Conference, Palm Springs, CA, vol. 2004-1560, American Institute of Aeronautics and Astronautics, 19-22 April 2004.

31. Oterkus E, Madenci E, Smeltzer III SS, Ambur DR. Non-linear analysis of bonded composite tubular lap joints. Proceedings of the AIAA/ASME/ASCE/AHS/ASC 46th Structures, Structural Dynamics, and Materials Conference, Austin, TX, vol. 2005-2380, American Institute of Aeronautics and Astronautics, 18-21 April 2005.

32. Smeltzer III SS, Lundgren E. Analytical and numerical results for an adhesively bonded joint subjected to pure bending. Proceedings of the AIAA/ASME/ASCE/AHS/ASC 47th Structures, Structural Dynamics, and Materials Conference, Newport, RI, vol. 2006-1960, American Institute of Aeronautics and Astronautics, 1-4 May 2006.

33. Bednarcyk EA, Zhang J, Collier CS, Bansal Y, Pindera MJ. Analysis tools for adhesively bonded composite joints, part 1: higher-order theory. AIAA Journal 2006; 44(1):171-180.

34. Hart-Smith LJ. Adhesive-bonded double-lap joints. NASA Contractor Report 112235, 1973.

35. Kilic B, Madenci E, Ambur DR. Global-local finite element analysis of bonded single-lap joints. 45th AIAA/ASME/ASCE/AHS/ASC Structures, Structrual Dynamics and Materials Conference, Palm Springs, CA, 19-22 April 2004; 1-13.

36. Zhang J, Collier C, Bansal Y, Bednarcyk BA, Pindera M-J. Analysis of adhesively bonded composite joints using a higher-order theory. 45th AIAA/ASME/ASCE/AHS/ASC Structures, Structrual Dynamics and Materials Conference, Palm Springs, CA, 19-22 April 2004; 1-16.

37. Gustafson PA, Bizard A, Waas AM. Dimensionless parameters in symmetric double lap joints: an orthotropic solution for thermomechanical loading. International Journal of Solids and Structures 2007; 44(17):5774-5795.

38. Davies GAO. Virtual Work in Structural Analysis. Wiley: New York, 1982.

39. Simulia, Inc. Abaqus User Manual v6.7. Electronic Version, 2007.

40. Cook RD, Malkus DS, Plesha ME. Concepts and Applications of Finite Element Analysis. Wiley: New York, 1989.

41. ASTM International. ASTM D3528-96 Standard Test Method for Strength Properties of Double Lap Shear Adhesive Joints by Tension Loading, ASTM International, 1996.

42. Herakovich CT. Mechanics of Fibrous Composites. Wiley Inc.: New York, 1998. 